\title{
Robust fault and icing diagnosis in unmanned aerial vehicles using LPV interval observers
}

\author{
Damiano Rotondo ${ }^{1,3,4 *}$ Andrea Cristofaro ${ }^{2}$, Tor Arne Johansen ${ }^{1}$, \\ Fatiha Nejjari ${ }^{3}$, Vicenç Puig ${ }^{3,4}$ \\ ${ }^{1}$ Center for Autonomous Marine Operations and Systems (AMOS), Department of Engineering Cybernetics, \\ Norwegian University of Science and Technology, Trondheim, Norway. \\ ${ }^{2}$ Scuola di Scienze e Tecnologie, Università di Camerino, Camerino (MC), Italy. \\ ${ }^{3}$ Advanced Control Systems (SAC), Universitat Politècnica de Catalunya (UPC), Rambla de Sant Nebridi, 11, Terrassa, \\ Spain. \\ ${ }^{4}$ Institut de Robotica i Informatica Industrial (IRI), UPC-CSIC Carrer de Llorens i Artigas, 4-6, Barcelona, Spain.
}

\begin{abstract}
SUMMARY
This paper proposes a linear parameter varying (LPV) interval unknown input observer (UIO) for the robust fault diagnosis of actuator faults and ice accretion in unmanned aerial vehicles (UAVs) described by an uncertain model. The proposed interval observer evaluates the set of values for the state which are compatible with the nominal fault-free and icing-free operation, and can be designed in such a way that some information about the nature of the unknown inputs affecting the system can be obtained, thus allowing the diagnosis to be performed. The proposed strategy has several advantages. First, the LPV paradigm allows taking into account operating point variations. Second, the noise rejection properties are enhanced by the presence of the integral term. Third, the interval estimation property guarantees the absence of false alarms. Linear matrix inequality (LMI)-based conditions for the analysis/design of these observers are provided in order to guarantee the interval estimation of the state and the boundedness of the estimation. The developed theory is supported by simulation results, obtained with the uncertain model of a Zagi Flying Wing UAV, which illustrate the strong appeal of the methodology for identifying correctly unexpected changes in the system dynamics due to actuator faults or icing. Copyright (c) 2018 John Wiley \& Sons, Ltd.
\end{abstract}

Received ...

KEY WORDS: Unknown input observers (UIOs), Linear parameter varying (LPV) systems, Icing diagnosis, Fault diagnosis, Unmanned aerial vehicles (UAVs), Uncertain systems, Interval observers

\footnotetext{
${ }^{*}$ Correspondence to: Damiano Rotondo, Institut de Robòtica i Informàtica Industrial (IRI), UPC-CSIC, Spain. Email: damiano.rotondo@yahoo.it

Copyright (c) 2018 John Wiley \& Sons, Ltd.
}

Prepared using rncauth.cls [Version: 2010/03/27 v2.00] 


\section{INTRODUCTION}

Feedback control systems are vulnerable to malfunctions in actuators, sensors or other system components, which may have catastrophic consequences, e.g. instability of the closed-loop system. For this reason, fault tolerant control techniques have been investigated widely in the last decades, with the aim of maintaining stability and acceptable performances in the event of faults [1]. As a consequence, the problem of detecting and identifying faults has become a hot topic of research, leading to the development of fault diagnosis techniques [2] with several proposed solutions, involving geometric [3], observer-based [4] and multiple model [5] approaches, among others.

Icing, i.e. the accretion of ice on the aircrafts' surfaces is one of the most critical faults affecting aviation safety [6]. The aerodynamic consequences of icing (an increase in drag and a decrease in lift) have a strong effect on the aircraft's performances, inducing a safety risk that can potentially lead to crashing [7]. In the case of small unmanned aerial vehicles (UAVs), some ice protection systems have been proposed recently in order to mitigate or eliminate the icing, based on heat conducting tapes [8] and electrically conductive carbon nanomaterial based coating for temperature control of UAV airfoil surfaces $[9,10]$. However, due to the large power consumption, fault/icing detection schemes [11] with fast and accurate responses are needed for assuring high efficiency. The approaches recently applied to icing detection in aircrafts and UAVs include multiple models [12,13], statistical methods [14], aerodynamic coefficient estimators [15] and environmental monitoring [16].

Unknown input observers (UIOs) are a special class of observers which allow estimating the state of a system independently of some unknown inputs [17]. UIOs are a very useful tool for achieving a successful fault detection and isolation [18], because they can be made insensitive to certain input space directions if some structural algebraic conditions on the system are fulfilled [19,20]. This property has been exploited in some recent works in order to perform fault/icing diagnosis. In [21], a UIO-based diagnosis scheme able to decouple icing effects from actuators or sensors faults was proposed for the longitudinal steady-state dynamics of a UAV. In [22], this approach was generalized to the linear 6-DOF motion model with coupled longitudinal/lateral dynamics, and integrated with a fault tolerant allocation scheme. A linear parameter varying (LPV) UIO-based icing diagnosis scheme has been presented in [23], with the main advantage of being consistent with the UAV dynamics for a wide range of operating conditions. Later, this work has been extended in [24], where an LPV proportional integral UIO has been used, with the advantage of being more robust against measurement noise.

All the aforementioned approaches share a common limitation, which is that they have been developed under the assumption of having a perfectly known model available for fault diagnosis purposes. However, it is well known that the presence of uncertainties coming from the mismatch 
between the model and the real system may impede the convergence of a classical observer to the exact value of the state $[25,26,27]$. In this situation, the use of interval observers is attractive because, under some assumptions, they can provide the set of admissible values for the state at each instant of time [28]. Unlike stochastic approaches, such as the Kalman filter [29], interval observers ignore any probability distribution at the sources of uncertainty, and assume that they are constrained in a known bounded set. Using this information, instead of a single trajectory for each state variable, the interval observer computes the lower and upper bounds, which are compatible with the uncertainty [30]. A successful framework for interval observer design is based on the monotone system theory, proposed at first by [31], and further investigated by [32, 33, 34].

The goal of this paper is to provide a framework for robust fault and icing diagnosis in UAVs, obtained by merging the theory of interval observers with the theory of UIOs. In contrast to [24], where the LPV UIO was obtained under the assumption that the model was perfectly known, in this paper an uncertain longitudinal model of the UAV motion is employed. From a theoretical point of view, the developed approach is an extension of the LPV interval UIO described in [35] to the proportional integral case. The inclusion of an integral action is needed in order to avoid the estimation error dynamics to be affected by the sensor noise derivative, which can take big values due to the high-frequency content of the noise signal. Simulation results, obtained with the uncertain model of a Zagi Flying Wing UAV, are used to show the effectiveness of the proposed approach.

The paper is structured as follows. Section 2 presents the notation and some preliminary background. Section 3 describes the proportional integral interval observer which solves the problem of interval state estimation without unknown input (detailing the green blocks in Fig. 1). Section 4 shows how the interval observer can be designed in order to behave as a UIO (detailing the violet blocks in Fig. 1). Section 5 introduces the nonlinear and quasi-LPV model of a UAV, the description of the icing effects and the application of the proposed UIO to robust fault/icing diagnosis (detailing the red blocks in Fig. 1). In Section 6, the proposed approach is illustrated using simulation results obtained with the uncertain model of a Zagi Flying Wing UAV. Finally, Section 7 summarizes the main conclusions.

\section{NOTATION AND BACKGROUND}

The set of (non-negative) real numbers will be denoted by $\mathbb{R}\left(\mathbb{R}_{+}\right)$. For a generic vector $x \in \mathbb{R}^{n_{x}}$, let us define $x^{+}=\max \{0, x\}$, where max denotes the elementwise maximum, $x^{-}=x^{+}-x$, and let us denote the vector of absolute values of all elements by $|x|=x^{+}+x^{-} . \mathscr{L}_{\infty}^{n_{u}}$ will denote the set of all signals $u$ such that $\|u\|_{\infty}=\sup \left\{|u(t)|, t \in \mathbb{R}_{+}\right\}<\infty$. Given a matrix $M \in \mathbb{R}^{m \times n}, H e\{M\}$ will be used as a shorthand notation for $M+M^{T}$. For two vectors $x_{1}, x_{2} \in \mathbb{R}^{n_{x}}$ or matrices $M_{1}, M_{2} \in \mathbb{R}^{m \times n}$, 


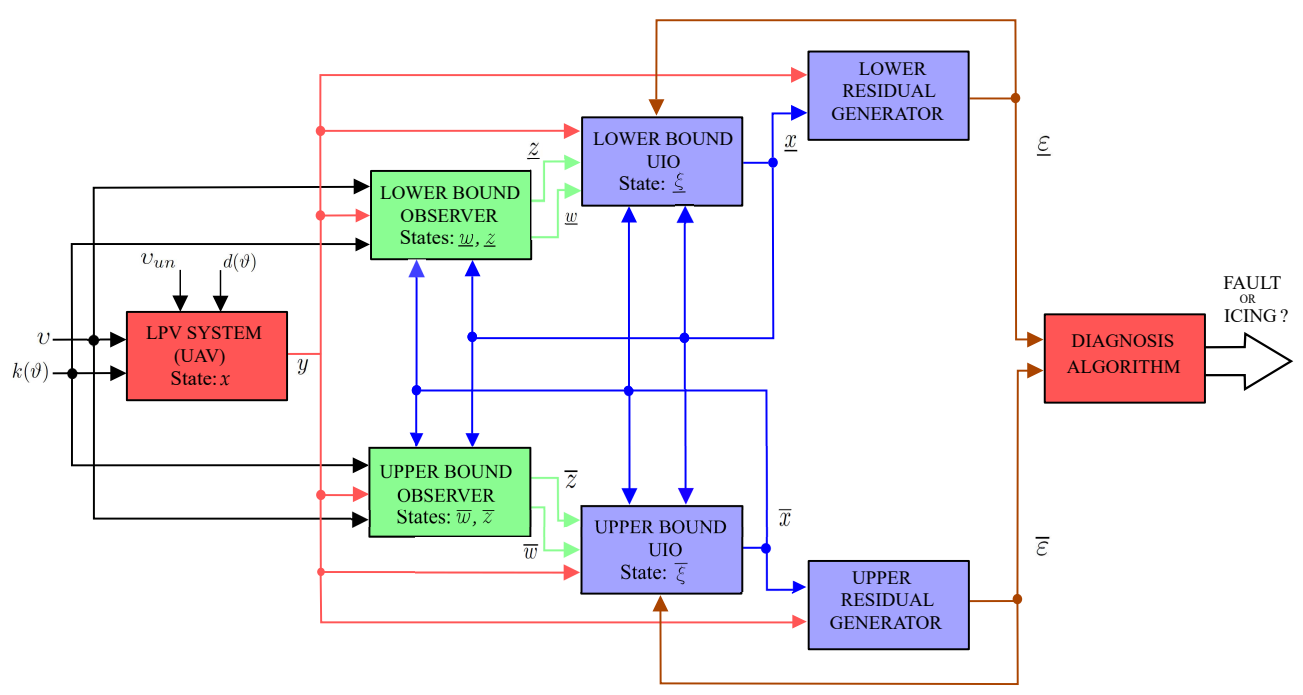

Figure 1. LPV UIO for the robust fault and icing diagnosis in UAVs.

the relations $x_{1} \leq x_{2}$ and $M_{1} \leq M_{2}$ are meant elementwise. The notation $M^{\dagger}$ denotes the MoorePenrose pseudoinverse [36] of the matrix $M \in \mathbb{R}^{m \times n}$. If $M \in \mathbb{R}^{n \times n}$ is symmetric, then $M \in \mathbb{S}^{n \times n}$. The notation $M \prec 0(M \succ 0)$ means that the matrix $M \in \mathbb{S}^{n \times n}$ is negative (positive) definite. If $M \in \mathbb{S}^{n \times n}$ is diagonal, then $M \in \mathbb{D}^{n \times n}$. If all the elements of a matrix $M \in \mathbb{R}^{n \times n}$ outside the main diagonal are nonnegative, then $M \in \mathbb{M}^{n \times n}$ will be called a Metzler matrix. The notation $\|M\|_{2}$ denotes the spectral norm of $M$, i.e. its largest singular value.

For a generic vector $x \in \mathbb{R}^{n_{x}}$, its $i$-th element will be denoted by $x^{(i)}$. For a given matrix $M \in \mathbb{R}^{m \times n}$ and a set of column indices $\mathscr{N}$, with $\mathscr{N}$ a subset of $\left\{1, \ldots, n_{x}\right\}$, the $i$-th column of $M$ will be denoted by $M^{(i)}$, while $M^{(\mathscr{N})}$ will denote the matrix obtained from $M$ by replacing all columns whose indices do not belong to $\mathscr{N}$ with zeros. Also, the notation $\Pi(M) x$ will denote the projection of $x$ onto the subspace generated by the columns of $M$. Given a set $\mathscr{S}$, the notation $\mathscr{P}(\mathscr{S})$ will denote the power set of $\mathscr{S}$, i.e. the set of all subsets of $\mathscr{S}$, including the empty set and $\mathscr{S}$ itself. In order to ease the notation, in many cases, the explicit dependence of the variables on time $t$ is omitted.

\section{INTERVAL STATE OBSERVATION WITHOUT UNKNOWN INPUT}

\subsection{Problem formulation}

As recalled in the introduction, interval observers evaluate the set of admissible values for the state at each time instant. In this section, the concept of interval observers will be extended to a proportional integral structure. The observer will compute lower and upper bounds for the state expressed in a time varying basis, i.e. transformed by a change of basis through a matrix function $R(\vartheta)$ which depends on a varying parameter vector $\vartheta \in \Theta$, containing exogenous variables, 
endogenous variables (e.g. states and/or inputs), or a combination of them, with $\Theta$ being a known closed and bounded set. This change of basis will be of paramount importance in order to perform a successful icing/fault diagnosis, as explained in the following sections. In general, the interval observer will require the knowledge of $\dot{\vartheta}$. However, as it will be discussed later, in the case of fault/icing diagnosis, this requirement can be relaxed.

Let us consider a continuous-time LPV system, as follows:

$$
\begin{aligned}
& \dot{x}=[A(\vartheta)+\Delta A(\vartheta)] x+[B(\vartheta)+\Delta B(\vartheta)] v+\left[B_{u n}(\vartheta)+\Delta B_{u n}(\vartheta)\right] v_{u n}+k(\vartheta)+d(\vartheta) \\
& y=x+v
\end{aligned}
$$

where $x$ is the state vector, $v$ is the known input (control action), $v_{u n}$ is the unknown input, $k(\vartheta)$ is a known term, $d(\vartheta)$ is an unknown disturbance, $y$ is the output vector and $v$ is the measurement noise.

Remark 1: The case where the output equation is given by $y=C x+v$ with $C$ full row rank matrix can be considered following the ideas presented in [35], although at the cost of increasing the mathematical complexity. In order to keep the formulation simple, such case will not be detailed in this paper. On the other hand, a theoretical challenge for the approach proposed in this paper is to consider the case in which the output matrix depends on the varying parameters, i.e. $y=C(\vartheta) x+v$. It is worth noting that, as long as the sensors' dynamics can be described by a state-space model with constant output and feedthrough matrices, i.e.:

$$
\begin{aligned}
& \dot{x}_{s}=A_{s}(\vartheta) x_{s}+B_{s}(\vartheta) x \\
& y=C_{s} x_{s}+D_{s} x
\end{aligned}
$$

then, the augmented system would satisfy:

$$
\begin{aligned}
& \left(\begin{array}{c}
\dot{x} \\
\dot{x}_{s}
\end{array}\right)=\left(\begin{array}{cc}
A(\vartheta)+\Delta A(\vartheta) & 0 \\
B_{s}(\vartheta) & A_{s}(\vartheta)
\end{array}\right)\left(\begin{array}{l}
x \\
x_{s}
\end{array}\right)+\left(\begin{array}{l}
* \\
0
\end{array}\right) \\
& y=\left(\begin{array}{ll}
D_{s} & C_{s}
\end{array}\right)\left(\begin{array}{ll}
x & x_{s}
\end{array}\right)^{T}
\end{aligned}
$$

where $*$ denotes terms depending on $v, v_{u n}, k(\vartheta)$ and $d(\vartheta)$, in such a way that the above-mentioned challenge would not exist. At the same time, a practical solution for the case where $y=C(\vartheta) x+v$ would be to postfilter the outputs, as suggested by [37].

Before stating the problem, let us introduce an assumption about the boundedness of disturbances, noise and uncertainties.

Assumption 1. The signal $v$ is such that $|v| \leq V$ for all $t \geq 0$ and some known $V \in \mathbb{R}^{n_{x}}$. Moreover, given an invertible and continuous matrix function $R(\vartheta) \in \mathbb{R}^{n_{x} \times n_{x}}$, there exist $\underline{d_{R}}(\vartheta), \overline{d_{R}}(\vartheta) \in$ $\mathscr{L}_{\infty}^{n_{x}}, \underline{\Delta A_{R}}(\vartheta), \overline{\Delta A_{R}}(\vartheta) \in \mathbb{R}^{n_{x} \times n_{x}}$ and $\underline{\Delta B_{R}}(\vartheta), \overline{\Delta B_{R}}(\vartheta) \in \mathbb{R}^{n_{x} \times n_{v}}$, with $\underline{\Delta A_{R}}(\vartheta), \underline{\Delta B_{R}}(\vartheta) \leq 0$ and 
$\overline{\Delta A_{R}}(\vartheta), \overline{\Delta B_{R}}(\vartheta) \geq 0$, such that for all $\vartheta \in \Theta$ :

$$
\begin{aligned}
& \underline{d_{R}}(\vartheta) \leq R(\vartheta) d(\vartheta) \leq \overline{d_{R}}(\vartheta) \\
& \underline{\Delta A_{R}}(\vartheta) \leq \Delta A_{R}(\vartheta)=R(\vartheta) \Delta A(\vartheta) R(\vartheta)^{-1} \leq \overline{\Delta A_{R}}(\vartheta) \\
& \underline{\Delta B_{R}}(\vartheta) \leq R(\vartheta) \Delta B(\vartheta) \leq \overline{\Delta B_{R}}(\vartheta)
\end{aligned}
$$

Remark 2: The assumption that $\underline{\Delta A_{R}}(\vartheta), \underline{\Delta B_{R}}(\vartheta) \leq 0$ and $\overline{\Delta A_{R}}(\vartheta), \overline{\Delta B_{R}}(\vartheta) \geq 0$ is not strictly needed, but allows keeping the mathematical formulation simple.

Problem 1: Given an invertible and continuous matrix function $R(\vartheta) \in \mathbb{R}^{n_{x} \times n_{x}}$, determine an LPV interval observer, which computes $\underline{x}$ and $\bar{x}$ such that:

$$
\underline{x_{R}}=R(\vartheta) \underline{x} \leq x_{R}=R(\vartheta) x \leq \overline{x_{R}}=R(\vartheta) \bar{x} \quad \forall t \geq 0
$$

with $\underline{x_{R}}, \overline{x_{R}} \in \mathscr{L}_{\infty}^{n_{x}}$, provided that:

$$
\begin{gathered}
\underline{x_{R}}(0) \leq x_{R}(0) \leq \overline{x_{R}}(0) \\
v_{u n}=0 \quad \forall t \geq 0
\end{gathered}
$$

and Assumption 1 holds.

\subsection{LPV proportional integral interval observer}

As discussed in [38, 39, 40], a limitation of purely proportional UIOs, as the one introduced in [35], is that the resulting error dynamics is affected by the noise derivative, which takes big values due to the high-frequency content of the noise signal. The introduction of an integral action allows overcoming this limitation. Hence, the LPV interval observer proposed in order to solve Problem 1 is given by two coupled subsystems, i.e. a lower bound observer, which provides $\underline{x}$, as follows:

$$
\begin{aligned}
& \underline{\dot{z}}=A(\vartheta) \underline{z}+B(\vartheta) v+k(\vartheta)+A(\vartheta) \underline{T}(\vartheta) \underline{w}-\underline{T}(\vartheta) \underline{w}+R(\vartheta)^{-1}\left(d_{R}(\vartheta)-|R(\vartheta) \underline{T}(\vartheta) \underline{K}(\vartheta)| V\right) \\
& +R(\vartheta)^{-1}\left[\underline{\Delta A_{R}}(\vartheta){\overline{x_{R}}}^{+}-\overline{\Delta A_{R}}(\vartheta) \underline{x_{R}}{ }^{-}+\underline{\Delta B_{R}}(\vartheta) v^{+}-\overline{\Delta B_{R}}(\vartheta) v^{-}\right] \\
& \underline{\dot{w}}=\underline{K}(\vartheta)(y-\underline{x}) \\
& \underline{x}=\underline{z}+\underline{T}(\vartheta) \underline{w}
\end{aligned}
$$

and an upper bound observer, which provides $\bar{x}$, as follows:

$$
\begin{aligned}
\dot{\bar{z}}= & A(\vartheta) \bar{z}+B(\vartheta) v+k(\vartheta)+A(\vartheta) \bar{T}(\vartheta) \bar{w}-\dot{\bar{T}}(\vartheta) \bar{w}+R(\vartheta)^{-1}\left(\overline{d_{R}}(\vartheta)+|R(\vartheta) \bar{T}(\vartheta) \bar{K}(\vartheta)| V\right) \\
& +R(\vartheta)^{-1}\left[\overline{\Delta A_{R}}(\vartheta){\overline{x_{R}}}^{+}-\underline{\Delta A_{R}(\vartheta) \underline{x_{R}}}{ }^{-}+\overline{\Delta B_{R}}(\vartheta) v^{+}-\underline{\Delta B_{R}}(\vartheta) v^{-}\right] \\
\dot{\bar{w}}= & \bar{K}(\vartheta)(y-\bar{x}) \\
\bar{x}= & \bar{z}+\bar{T}(\vartheta) \bar{w}
\end{aligned}
$$


where $\underline{T}(\vartheta), \bar{T}(\vartheta), \underline{K}(\vartheta), \bar{K}(\vartheta) \in \mathbb{R}^{n_{x} \times n_{x}}$ are the interval observer gains, while $\underline{\dot{T}}(\vartheta)$ and $\dot{\bar{T}}(\vartheta)$ can be obtained from $\underline{T}(\vartheta)$ and $\bar{T}(\vartheta)$ by differentiating each element with respect to time ${ }^{\dagger}$.

It can be seen that the estimated bounds calculated as (15) and (18) are an appropriate combination of the proportional action, given by (13) and (16), and the integral action, given by (14) and (17).

Remark 3: The terms post-multiplying $R(\vartheta)^{-1}$ in (13) and (16) take into account the known bounds of disturbances, noise and uncertainties, in order to provide an interval estimation of the state transformed through the parameter varying matrix function $R(\vartheta)$.

The following theorem provides the conditions which should be satisfied by the gains $\underline{K}(\vartheta)$, $\bar{K}(\vartheta), \underline{T}(\vartheta)$ and $\bar{T}(\vartheta)$ to ensure an interval estimation of $x_{R}$ and the boundedness of $\underline{x_{R}}, \overline{x_{R}}$, as specified in Problem 1.

\section{Theorem 1}

Let Assumption 1 be satisfied, $x \in \mathscr{L}_{\infty}^{n_{x}}, v \in \mathscr{L}_{\infty}^{n_{v}}, k \in \mathscr{L}_{\infty}^{n_{x}}$, the matrix function $R(\vartheta)$ be invertible, and the proportional integral interval observer be given by (13)-(18). Then, if there exist matrix functions $\underline{K}(\vartheta), \bar{K}(\vartheta), \underline{T}(\vartheta), \bar{T}(\vartheta) \in \mathbb{R}^{n_{x} \times n_{x}}$ such that:

$$
\begin{aligned}
& \underline{F}(\vartheta)=[R(\vartheta) A(\vartheta)-R(\vartheta) \underline{T}(\vartheta) \underline{K}(\vartheta)+\dot{R}(\vartheta)] R(\vartheta)^{-1} \in \mathbb{M}^{n_{x} \times n_{x}} \\
& \bar{F}(\vartheta)=[R(\vartheta) A(\vartheta)-R(\vartheta) \bar{T}(\vartheta) \bar{K}(\vartheta)+\dot{R}(\vartheta)] R(\vartheta)^{-1} \in \mathbb{M}^{n_{x} \times n_{x}}
\end{aligned}
$$

the relation (10) is satisfied provided that (11)-(12) hold.

In addition, if there exist $P, Q \in \mathbb{S}^{2 n_{x} \times 2 n_{x}}, P, Q \succ 0$ and constants $\varepsilon_{1}, \varepsilon_{2}, \gamma>0$ such that the following matrix inequality is verified:

$$
\Phi(\vartheta)=\left(\begin{array}{cc}
G(\vartheta)^{T} P+P G(\vartheta)+\left(\varepsilon_{1}+\varepsilon_{2}\right) P+Q+\gamma \eta(\vartheta)^{2} I_{2 n_{x}} & 0 \\
0 & \varepsilon_{1}^{-1} P-\gamma I_{2 n_{x}}
\end{array}\right) \preceq 0
$$

where:

$$
\begin{aligned}
& \eta(\vartheta)=2\left(\left\|\overline{\Delta A_{R}}(\vartheta)\right\|_{2}+\left\|\underline{\Delta A_{R}}(\vartheta)\right\|_{2}\right) \\
& G(\vartheta)=\left(\begin{array}{cc}
\underline{F}(\vartheta) & 0 \\
0 & \bar{F}(\vartheta)+\overline{\Delta A_{R}}(\vartheta)
\end{array}\right)
\end{aligned}
$$

then $\underline{x_{R}}, \overline{x_{R}} \in \mathscr{L}_{\infty}^{n_{x}}$.

Remark 4: The theorem statement consists of two parts. Eqs. (19)-(20) guarantee that, at each instant of time, the true state of the LPV system described by (1)-(2) will lie inside the region defined by the lower and upper estimates. On the other hand, the feasibility of the matrix inequality (21)

\footnotetext{
${ }^{\dagger}$ In general, $\underline{T}$ and $\dot{\bar{T}}$ will depend also on $\dot{\vartheta}$. However, since an augmented varying parameter vector made up by $\vartheta$ and $\dot{\vartheta}$ could be considered for further reasoning, the dependence of a matrix on $\dot{\vartheta}$ will be left implicit to ease the notation.
} 
ensures that such estimates will remain bounded, i.e. they will not diverge. Also in this case, $\dot{R}(\vartheta)$ can be obtained from $R(\vartheta)$ by differentiating each of its elements with respect to time.

Proof of Theorem 1: Let us consider the dynamics of the interval estimation errors $\underline{e}=$ $R(\vartheta)(x-\underline{x})=x_{R}-\underline{x_{R}}$ and $\bar{e}=R(\vartheta)(\bar{x}-x)=\overline{x_{R}}-x_{R}$ which, taking into account (1)-(2) and (13)(18), become:

$$
\begin{aligned}
& \dot{\dot{e}}=\underline{F}(\vartheta) \underline{e}+R(\vartheta)\left(B_{u n}(\vartheta)+\Delta B_{u n}(\vartheta)\right) v_{u n}+\sum_{i=1}^{4} \underline{\Phi_{i}} \\
& \dot{\bar{e}}=\bar{F}(\vartheta) \bar{e}-R(\vartheta)\left(B_{u n}(\vartheta)+\Delta B_{u n}(\vartheta)\right) v_{u n}+\sum_{i=1}^{4} \overline{\Phi_{i}}
\end{aligned}
$$

where $\underline{F}(\vartheta)$ and $\bar{F}(\vartheta)$ are defined as in (19)-(20) and $\underline{\varpi_{i}}, i=1, \ldots, 4$, are given by (similar expressions hold for $\left.\overline{\varpi_{i}}, i=1, \ldots, 4\right)$ :

$$
\begin{aligned}
& \underline{\varpi_{1}}=|R(\vartheta) \underline{T}(\vartheta) \underline{K}(\vartheta)| V-R(\vartheta) \underline{T}(\vartheta) \underline{K}(\vartheta) v \\
& \underline{\varpi_{2}}=R(\vartheta) d(\vartheta)-\underline{d_{R}}(\vartheta) \\
& \underline{\varpi_{3}}=\Delta A_{R}(\vartheta) x_{R}+\overline{\Delta A_{R}}(\vartheta) \underline{x_{R}}{ }^{-}-\underline{\Delta A_{R}}(\vartheta) \overline{x_{R}}{ }^{+} \\
& \underline{\varpi_{4}}=R(\vartheta) \Delta B(\vartheta) v+\overline{\Delta B_{R}}(\vartheta) v^{-}-\underline{\Delta B_{R}}(\vartheta) v^{+}
\end{aligned}
$$

When (12) holds, since $\underline{F}(\vartheta), \bar{F}(\vartheta) \in \mathbb{M}^{n_{x} \times n_{x}}$, then any solution of (24)-(25) is elementwise nonnegative for all $t \geq 0$, i.e. (10), provided that $\underline{e}(0) \geq 0, \bar{e}(0) \geq 0, \underline{\Phi_{i}} \geq 0$ and $\overline{\Phi_{i}} \geq 0 \forall t \geq 0$, $\forall i=1,2,3,4$ [41]. $\underline{e}(0) \geq 0$ and $\bar{e}(0) \geq 0$ hold due to (11). The terms $\underline{\varpi_{i}}, \overline{\bar{\omega}_{i}}, i=1,2$, are nonnegative $\forall t \geq 0$ due to Assumption 1 (see (7)). On the other hand, $\underline{\Phi_{3}}, \overline{\omega_{3}}$ remain nonnegative as long as (10) holds, according to the results in [26] and Assumption 1 (see (8)). (10) holds for $t=0$, due to $\underline{e}(0) \geq 0, \bar{e}(0) \geq 0$, and (10) is preserved $\forall t \geq 0$ by induction, as long as $\underline{\Phi_{4}}, \overline{\Phi_{4}}$ remain nonnegative too. Indeed, also $\underline{\varpi_{4}}, \overline{\varpi_{4}}$ remain nonnegative because of the results in [26] and Assumption 1 (see (9)).

Let us show that the variables $\underline{x_{R}}$ and $\overline{x_{R}}$ stay bounded $\forall t \geq 0$. For this purpose, let us rewrite the equations that rule the dynamics of $\underline{x_{R}}$ and $\overline{x_{R}}$ as:

$$
\dot{\xi}=G(\vartheta) \xi+\phi(\xi)+\delta
$$

where $\xi=\left(\underline{x_{R}}, \overline{x_{R}}\right)^{T}, \phi(\xi)=\left(\underline{f}\left(\underline{x_{R}}, \overline{x_{R}}\right), \bar{f}\left(\underline{x_{R}}, \overline{x_{R}}\right)\right)^{T}$ and $\delta=(\underline{\delta}, \bar{\delta})^{T}$, with (similar expressions hold for $\bar{f}\left(\underline{x_{R}}, \overline{x_{R}}\right)$ and $\left.\bar{\delta}\right)$ :

$$
\begin{aligned}
& \underline{f}\left(\underline{x_{R}}, \overline{x_{R}}\right)=\underline{\Delta A_{R}}(\vartheta){\overline{x_{R}}}^{+}-\overline{\Delta A_{R}}(\vartheta) \underline{x_{R}}{ }^{-} \\
& \underline{\delta}=R(\vartheta) \underline{T}(\vartheta) \underline{K}(\vartheta) y-|R(\vartheta) \underline{T}(\vartheta) \underline{K}(\vartheta)| V+R(\vartheta) B(\vartheta) v \\
& \quad+R(\vartheta) k(\vartheta)+\underline{d_{R}}(\vartheta)+\underline{\Delta B_{R}}(\vartheta) v^{+}-\overline{\Delta B_{R}}(\vartheta) v^{-}
\end{aligned}
$$

Notably, for all $\vartheta \in \Theta$ :

$$
|\phi(\xi)| \leq \eta(\vartheta)|\xi|
$$


with $\eta(\vartheta)$ given by (22), while the inputs $\underline{\delta}, \bar{\delta}$ are bounded by Assumption 1 and the fact that $x \in \mathscr{L}_{\infty}^{n_{x}}, v \in \mathscr{L}_{\infty}^{n_{v}}, k \in \mathscr{L}_{\infty}^{n_{x}}$. Then, by considering a Lyapunov function $V=\xi^{T} P \xi$ and through input to state stability reasoning [42], it follows that if (21) holds, then $x_{R}, \overline{x_{R}} \in \mathscr{L}_{\infty}^{n_{x}}$.

Remark 5: For illustrative purposes, let us consider a lower bound proportional observer given by:

$$
\begin{aligned}
\dot{z} & =A(\vartheta) \underline{z}+B(\vartheta) v+k(\vartheta)+A(\vartheta) T(\vartheta) y-\dot{T}(\vartheta) y+R(\vartheta)^{-1}\left(\underline{d_{T}}(\vartheta)-|R(\vartheta) T(\vartheta)| \dot{v}_{\max }\right) \\
& +R(\vartheta)^{-1}\left[\underline{\Delta A_{T}}(\vartheta){\overline{x_{R}}}^{+}-\overline{\Delta A_{T}}(\vartheta) \underline{x_{R}}{ }^{-}+\underline{\Delta B_{T}}(\vartheta) v^{+}-\overline{\Delta B_{T}}(\vartheta) v^{-}\right]-T(\vartheta)[A(\vartheta) \underline{x}+B(\vartheta) v+k(\vartheta)] \\
\underline{x} & =\underline{z}+T(\vartheta) y
\end{aligned}
$$

where $\dot{v}_{\max }$ is a known bound on the noise derivative $\dot{v}$. It can be shown that, under conditions similar to those of Assumption 1, the estimation error with $v_{u n}=0$ obeys:

$$
\underline{\dot{e}}=\underline{F_{T}}(\vartheta) \underline{e}+\sum_{i=1}^{4} \underline{\varpi_{T, i}}
$$

with:

$$
\underline{F_{T}}(\vartheta)=[R(\vartheta)(I-T(\vartheta)) A(\vartheta)+\dot{R}(\vartheta)] R(\vartheta)^{-1}
$$

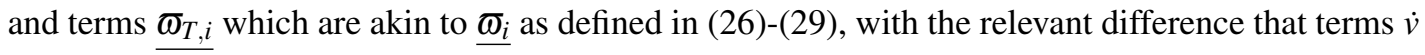
and $\dot{v}_{\text {max }}$ appear instead of $v$ and $V$, which would lead to very conservative estimated bounds for the state.

\subsection{Design conditions}

Given the matrix functions $\underline{K}(\vartheta), \bar{K}(\vartheta), \underline{T}(\vartheta), \bar{T}(\vartheta)$ (interval observer gains), the conditions provided by Theorem 1, i.e. (19)-(21), allow analysing whether or not the observer (13)-(18) will provide a bounded interval estimation of the state. At the expense of introducing some conservativeness, it is possible to derive conditions for performing the design, i.e. for the case where $\underline{K}(\vartheta), \bar{K}(\vartheta), \underline{T}(\vartheta), \bar{T}(\vartheta)$ are not given, such that they are obtained as part of the solution of the LMIs. This can be done using the following corollary.

\section{Corollary 1}

Let Assumption 1 be satisfied, $x \in \mathscr{L}_{\infty}^{n_{x}}, v \in \mathscr{L}_{\infty}^{n_{v}}, k \in \mathscr{L}_{\infty}^{n_{x}}$, and the matrix function $R(\vartheta)$ be invertible. Also, let us assume that there exist an elementwise nonnegative matrix:

$$
P=\left(\begin{array}{cc}
\underline{P} & 0 \\
0 & \bar{P}
\end{array}\right)
$$

with $\underline{P}, \bar{P} \in \mathbb{S}^{n_{x} \times n_{x}}, \underline{P}, \bar{P} \succ 0$, a matrix function:

$$
W(\vartheta)=\left(\begin{array}{cc}
\underline{W}(\vartheta) & 0 \\
0 & \bar{W}(\vartheta)
\end{array}\right)
$$


with $\underline{W}(\vartheta), \bar{W}(\vartheta) \in \mathbb{R}^{n_{x} \times n_{x}}$, a matrix $Q \in \mathbb{S}^{2 n_{x} \times 2 n_{x}}$, a sufficiently large matrix function $\Sigma(\vartheta) \in$ $\mathbb{D}_{+}^{2 n_{x} \times 2 n_{x}}$ and constants $\varepsilon_{1}, \varepsilon_{2}, \gamma>0$ such that:

$$
\begin{gathered}
\left(\begin{array}{cc}
H e\{P \Xi(\vartheta)-W(\vartheta) \Upsilon(\vartheta)\}+\left(\varepsilon_{1}+\varepsilon_{2}\right) P+Q+\gamma \eta(\vartheta)^{2} I_{2 n_{x}} & 0 \\
0 & \varepsilon_{1}^{-1} P-\gamma I_{2 n_{x}}
\end{array}\right) \preceq 0 \\
P\left(\begin{array}{cc}
{[R(\vartheta) A(\vartheta)+\dot{R}(\vartheta)] R(\vartheta)^{-1}} & 0 \\
0 & {[R(\vartheta) A(\vartheta)+\dot{R}(\vartheta)] R(\vartheta)^{-1}}
\end{array}\right)-W(\vartheta) \Upsilon(\vartheta)+P \Sigma(\vartheta) \geq 0
\end{gathered}
$$

with $\eta(\vartheta)$ defined as in (22) and:

$$
\begin{gathered}
\Xi(\vartheta)=\left(\begin{array}{cc}
R(\vartheta) A(\vartheta) R(\vartheta)^{-1}+\dot{R}(\vartheta) R(\vartheta)^{-1} & 0 \\
0 & R(\vartheta) A(\vartheta) R(\vartheta)^{-1}+\dot{R}(\vartheta) R(\vartheta)^{-1}+\overline{\Delta A_{R}}(\vartheta)
\end{array}\right) \\
\Upsilon(\vartheta)=\left(\begin{array}{cc}
C R(\vartheta)^{-1} & 0 \\
0 & C R(\vartheta)^{-1}
\end{array}\right)
\end{gathered}
$$

Then, the proportional integral interval observer (13)-(18) with matrices $\underline{K}(\vartheta), \bar{K}(\vartheta), \underline{T}(\vartheta), \bar{T}(\vartheta)$ satisfying:

$$
\left(\begin{array}{cc}
\underline{T}(\vartheta) \underline{K}(\vartheta) & 0 \\
0 & \bar{T}(\vartheta) \bar{K}(\vartheta)
\end{array}\right)=\left(\begin{array}{cc}
R(\vartheta) & 0 \\
0 & R(\vartheta)
\end{array}\right)^{-1} P^{-1} W(\vartheta)
$$

is such that the relation (10) holds provided that (11)-(12) are satisfied, with $\underline{x_{R}}, \overline{x_{R}} \in \mathscr{L}_{\infty}^{n_{x}}$.

Proof of Corollary 1: The matrix inequality (31) can be obtained easily from (21) through the change of variables $W(\vartheta)=\operatorname{diag}(\underline{P} R(\vartheta) \underline{T}(\vartheta) \underline{K}(\vartheta), \bar{P} R(\vartheta) \bar{T}(\vartheta) \bar{K}(\vartheta))$, which explains why $\underline{K}(\vartheta), \bar{K}(\vartheta), \underline{T}(\vartheta), \bar{T}(\vartheta)$ are calculated as (33). On the other hand, (32) corresponds to the verification of the Metzler property (19)-(20) [25].

Remark 6: It must be pointed out that both Theorem 1 and Corollary 1 rely on the satisfaction of infinite conditions. However, this difficulty can be overcome by gridding $\Theta$ using $N$ points $\vartheta_{i}$, $i=1, \ldots, N$. Then, once $\varepsilon_{1}$ and $\varepsilon_{2}$ have been chosen, the analysis/design problem reduces to finding a feasible solution of a set of LMIs, which can be done efficiently using available solvers, e.g. YALMIP/SeDuMi $[43,44]$. By relying on the gridding approach, the theoretical properties would be guaranteed only for $\vartheta=\vartheta_{i}, i=1, \ldots, N$, i.e. at the gridding points. However, from a practical point of view, it is reasonable to assume that if the gridding of $\Theta$ is dense enough, then they would still hold at operating points different from the gridding ones. A deep theoretical study of this fact is possible using the results developed by [45].

Remark 7: An alternative approach for calculating the matrices $\underline{F}(\vartheta)$ and $\bar{F}(\vartheta)$ consists in designing them to obtain boundedness of the estimated bounds, and then performing a similarity transformation such that in the new coordinates the resulting matrices are Metzler. The conditions of existence of such transformation can be derived following the reasoning in [34]. 


\section{INTERVAL UNKNOWN INPUT OBSERVER}

\subsection{Problem formulation}

The change of basis performed using the parameter varying matrix function $R(\vartheta)$ is relevant to obtain the decoupling between the effects of the unknown inputs $v_{u n}$ and solve the problem of unknown input observation. In this way, it will be possible to detect the presence of unknown inputs acting on the system, as well as to identify their nature (isolation). Before stating the problem, let us introduce an additional assumption concerning the boundedness of signals and uncertainties related to the unknown inputs.

Assumption 2. The signal $v_{u n}$ is such that:

$$
\underline{v_{u n}} \leq v_{u n} \leq \overline{v_{u n}}
$$

with $\underline{v_{u n}} \leq 0$ and $\overline{v_{u n}} \geq 0, \underline{v_{u n}}, \overline{v_{u n}} \in \mathscr{L}_{\infty}^{n_{v}}$. Moreover, given an invertible and continuous matrix function $R(\vartheta) \in \mathbb{R}^{n_{x} \times n_{x}}$, there exist $\Delta B_{u n, R}(\vartheta), \overline{\Delta B_{u n, R}}(\vartheta) \in \mathbb{R}^{n_{x} \times n_{\text {un }}}$, with $\Delta B_{u n, R}(\vartheta) \leq 0$, $\overline{\Delta B_{u n, R}}(\vartheta) \geq 0$, such that for all $\vartheta \in \Theta$ :

$$
\underline{\Delta B_{u n, R}}(\vartheta) \leq R(\vartheta) \Delta B_{u n}(\vartheta) \leq \overline{\Delta B_{u n, R}}(\vartheta)
$$

Problem 2. Given $S \in \mathbb{R}^{n_{x} \times n_{v_{u n}}}$ full column rank, such that there exists an invertible matrix function $R(\vartheta) \in \mathbb{R}^{n_{x} \times n_{x}}$ for which the following condition holds:

$$
R(\vartheta) B_{u n}(\vartheta)=S \quad \forall \vartheta \in \Theta
$$

and provided that (11) and Assumptions 1-2 hold, determine an LPV proportional integral interval unknown input observer which, in addition to solve Problem 1, satisfies:

$$
\begin{aligned}
& v_{u n}^{(j)}=0 \Rightarrow \Pi\left(S^{(j)}\right) \underline{\varepsilon} \geq 0 \wedge \Pi\left(S^{(j)}\right) \bar{\varepsilon} \geq 0 \\
& \Pi\left(S^{(j)}\right) \underline{\varepsilon}<0 \vee \Pi\left(S^{(j)}\right) \bar{\varepsilon}<0 \quad \Rightarrow \quad v_{u n}^{(j)} \neq 0
\end{aligned}
$$

where $\underline{\varepsilon}$ and $\bar{\varepsilon}$ are evaluable signals that can be used as unknown input isolation signals, and are given by:

$$
\begin{aligned}
& \underline{\varepsilon}=R(\vartheta)(y-\underline{x})-|R(\vartheta)| V \\
& \bar{\varepsilon}=R(\vartheta)(\bar{x}-y)-|R(\vartheta)| V
\end{aligned}
$$

In other words, Problem 2 concerns the isolation of faults, which can be represented by the unknown input $v_{u n}$ in (1). The idea consists in assigning different directions of residuals for each element of the vector $v_{u n}$, and choosing the interval observer in order to guarantee that, if the component of at least one value between $\underline{\varepsilon}$ and $\bar{\varepsilon}$ along the direction specified by the $j$-th column of 
the matrix $S$ becomes negative, then the $j$-th element of the vector $v_{u n}$ must be necessarily different from zero, which allows detecting and isolating the unknown input.

Remark 8: Note that the values $\underline{\varepsilon}=0$ and $\bar{\varepsilon}=0$ can be interpreted as thresholds [46] that the residuals will not exceed as long as the unknown inputs are not acting on the system. Due to the use of an interval observer formulation, the proposed approach is naturally robust against uncertainties and noise, as long as they satisfy Assumptions 1-2. However, defining a residual evaluation function over a finite time window, as discussed deeply in [47, 48], could help in increasing the overall robustness when the proposed approach is applied under conditions for which Assumptions 1-2 do not hold strictly.

\subsection{LPV proportional integral UIO}

Looking at (24)-(25), and recalling (36), it is evident that when $\Delta B_{u n}(\vartheta)=0$, in order to achieve this property, the columns of $S$ should correspond to eigenvectors of the matrices $\underline{F}(\vartheta), \bar{F}(\vartheta)$, and the terms $\underline{\varpi}_{i}, \bar{\varpi}_{i}$ should maintain nonnegativity despite a possible change in the sign of $\underline{\varepsilon}$ and/or $\bar{\varepsilon}$. This last property, which is not necessary for fault detection, but is fundamental to achieve fault isolation, requires a slight modification of the interval observer structure provided in (13)-(18). On the other hand, a further modification of (13)-(18) is performed to embed the term $R(\vartheta) \Delta B_{u n}(\vartheta) v_{u n}$ into nonnegative terms that will be referred to as $\bar{\varpi}_{5}$ and $\overline{\varpi_{5}}$.

The following LPV proportional integral interval unknown input observer is proposed to solve Problem 2:

$$
\begin{aligned}
& \underline{\dot{\xi}}=\underline{\dot{z}}+A(\vartheta)(\underline{\xi}-\underline{z})+R(\vartheta)^{-1} \sum_{i=1}^{n_{x}}\left\{\frac{1-\operatorname{sign}\left(\underline{\varepsilon}^{(i)}\right)}{2} \overline{\Delta A_{R}^{(i)}}(\vartheta)\left(\underline{x}_{R}^{(i)}{ }^{-}-\underline{x}_{R}^{(i)}\right)\right. \\
& \left.+\frac{1-\operatorname{sign}\left(\bar{\varepsilon}^{(i)}\right)}{2} \underline{\Delta A_{R}^{(i)}}(\vartheta)\left(\tilde{x}_{R}^{(i)}{ }^{+}-{\overline{x_{R}}}^{(i)^{+}}\right)\right\}+R(\vartheta)^{-1}\left[\underline{\Delta B_{u n, R}}(\vartheta) \overline{v_{u n}}+-\overline{\Delta B_{u n, R}}(\vartheta) \underline{v_{u n}}{ }^{-}\right] \\
& \underline{x}=\underline{\xi}+\underline{T}(\vartheta) \underline{w} \\
& \dot{\bar{\xi}}=\dot{\bar{z}}+A(\vartheta)(\bar{\xi}-\bar{z})+R(\vartheta)^{-1} \sum_{i=1}^{n_{x}}\left\{\frac{1-\operatorname{sign}\left(\bar{\varepsilon}^{(i)}\right)}{2} \overline{\Delta A_{R}^{(i)}}(\vartheta)\left(\tilde{x}_{R}^{(i)}{ }^{+}-{\overline{x_{R}}}^{(i)}{ }^{+}\right)\right. \\
& \left.+\frac{1-\operatorname{sign}\left(\underline{\varepsilon}^{(i)}\right)}{2} \underline{\Delta A_{R}^{(i)}}(\vartheta)\left(\underline{x}_{R}^{(i)^{-}}-x_{R}^{(i)}{ }^{-}\right)\right\}+R(\vartheta)^{-1}\left[\overline{\Delta B_{u n, R}}(\vartheta){\overline{v_{u n}}}^{+}-\underline{\Delta B_{u n, R}(\vartheta) \underline{v_{u n}}}{ }^{-}\right] \\
& \bar{x}=\bar{\xi}+\bar{T}(\vartheta) \bar{w}
\end{aligned}
$$

where $\underline{z}, \underline{w}, \dot{\bar{z}}, \bar{w}, \underline{\varepsilon}$ and $\bar{\varepsilon}$ are given by (13)-(14), (16)-(17) and (39)-(40), and:

$$
\begin{aligned}
& \underset{\sim}{x_{R}}=R(\vartheta) y-|R(\vartheta)| V \\
& \tilde{x}_{R}=R(\vartheta) y+|R(\vartheta)| V
\end{aligned}
$$


The following lemma provides the conditions which should be satisfied by the gains $\underline{K}(\vartheta), \bar{K}(\vartheta), \underline{T}(\vartheta)$ and $\bar{T}(\vartheta)$ in order to ensure an interval estimation of $x_{R}$ and the boundedness of $\underline{x_{R}}, \overline{x_{R}}$, as specified in Problem 1.

\section{Lemma 1}

Let Assumptions 1-2 be satisfied, $x \in \mathscr{L}_{\infty}^{n_{x}}, v \in \mathscr{L}_{\infty}^{n_{v}}, k \in \mathscr{L}_{\infty}^{n_{x}}$, the matrix $R(\vartheta)$ be invertible, and the proportional integral interval unknown input observer be given by (13)-(14), (16)-(17) and (41)(44). Then, if there exist matrix functions $\underline{K}(\vartheta), \bar{K}(\vartheta), \underline{T}(\vartheta), \bar{T}(\vartheta) \in \mathbb{R}^{n_{x} \times n_{x}}$ such that $\underline{F}(\vartheta), \bar{F}(\vartheta)$, defined as in (19)-(20) are Metzler, the relation (10) is satisfied provided that (11)-(12) hold.

In addition, if there exist $P, Q \in \mathbb{S}^{2 n_{x} \times 2 n_{x}}, P, Q \succ 0$ and constants $\varepsilon_{1}, \varepsilon_{2}, \gamma>0$ such that the following matrix inequality is verified $\forall \mathscr{S}_{1}, \mathscr{S}_{2} \in \mathscr{P}\left(\left\{1, \ldots, n_{x}\right\}\right)$ :

$$
\Phi\left(\vartheta, \mathscr{S}_{1}, \mathscr{S}_{2}\right)=\left(\begin{array}{cc}
G\left(\vartheta, \mathscr{S}_{2}\right)^{T} P+P G\left(\vartheta, \mathscr{S}_{2}\right)+\left(\varepsilon_{1}+\varepsilon_{2}\right) P+Q+\gamma \eta\left(\vartheta, \mathscr{S}_{1}, \mathscr{S}_{2}\right)^{2} I_{2 n_{x}} & 0 \\
0 & \varepsilon_{1}^{-1} P-\gamma I_{2 n_{x}}
\end{array}\right) \preceq 0
$$

where:

$$
\begin{gathered}
\eta\left(\vartheta, \mathscr{S}_{1}, \mathscr{S}_{2}\right)=\left\|\overline{\Delta A_{R}^{\mathscr{S}_{1}}(\vartheta)}\right\|_{2}+\left\|\underline{\Delta A_{R}^{\mathscr{S}_{1}}(\vartheta) \|_{2}}+\right\| \overline{\Delta A_{R}^{\mathscr{S}_{2}}}(\vartheta)\left\|_{2}+\right\| \underline{\Delta A_{R}^{\mathscr{S}_{2}}(\vartheta) \|_{2}} \\
G\left(\vartheta, \mathscr{S}_{2}\right)=\left(\begin{array}{cc}
\underline{F}(\vartheta) & 0 \\
0 & \bar{F}(\vartheta)+\overline{\Delta A_{R}^{\mathscr{S}_{2}}(\vartheta)}
\end{array}\right)
\end{gathered}
$$

then $\underline{x_{R}}, \overline{x_{R}} \in \mathscr{L}_{\infty}^{n_{x}}$.

Similarly to Theorem 1, the matrix inequality (45) is needed to ensure that the lower and upper estimates provided by the interval observer will remain bounded despite the modifications in the structure of the observer due to changes in the signs of $\underline{\varepsilon}^{(i)}, \bar{\varepsilon}^{(i)}, i=1, \ldots, n_{x}$. This fact will be further detailed in the proof of Lemma 1.

Proof of Lemma 1: By using the interval unknown input observer (13)-(14), (16)-(17) and (41)(44), the dynamics of the interval estimation errors $\underline{e}, \bar{e}$ follow:

$$
\begin{aligned}
& \underline{\dot{e}}=\underline{F}(\vartheta) \underline{e}+R(\vartheta) B_{u n}(\vartheta) v_{u n}+\sum_{i=1}^{5} \frac{\Phi_{i}}{} \\
& \dot{\bar{e}}=\bar{F}(\vartheta) \bar{e}-R(\vartheta) B_{u n}(\vartheta) v_{u n}+\sum_{i=1}^{5} \overline{\varpi_{i}}
\end{aligned}
$$

where $\bar{\Phi}_{i}, \overline{\omega_{i}}, i=1,2,4$, are as in the proof of Theorem 1 , and (similar expressions hold for $\overline{\varpi_{3}}$ and $\left.\overline{\omega_{5}}\right)$ :

$$
\begin{aligned}
\underline{\omega_{3}} & =\sum_{i=1}^{n_{x}}\left[\Delta A_{R}^{(i)}(\vartheta) x_{R}^{(i)}+\overline{\Delta A_{R}^{(i)}}(\vartheta) \underline{x_{R}}{ }^{(i)^{-}}-\underline{\Delta A_{R}^{(i)}}(\vartheta){\overline{x_{R}}}^{(i)^{+}}+\frac{1-\operatorname{sign}\left(\underline{\varepsilon}^{(i)}\right)}{2} \overline{\Delta A_{R}^{(i)}}(\vartheta)\left(\underline{x}_{R}^{(i)}{ }^{-}-\underline{x}_{R}^{(i)}{ }^{-}\right)\right] \\
& -\sum_{i=1}^{n_{x}} \frac{1-\operatorname{sign}\left(\bar{\varepsilon}^{(i)}\right)}{2} \underline{\Delta A_{R}^{(i)}(\vartheta)\left(\tilde{x}_{R}^{(i)}{ }^{+}-{\overline{x_{R}}}^{(i)}{ }^{+}\right)} \\
\underline{\bar{\omega}_{5}} & =R(\vartheta) \Delta B_{u n}(\vartheta) v_{u n}+\overline{\Delta B_{u n, R}}(\vartheta) \underline{v_{u n}}{ }^{-}-\underline{\Delta B_{u n, R}}(\vartheta){\overline{v_{u n}}}^{+}
\end{aligned}
$$


As it has already been discussed, the terms $\bar{\varpi}_{i}, \overline{\varpi_{i}}, i=1,2,4$ are nonnegative due to Assumption 1 and (11). Let us show that $\underline{\varpi_{3}} \geq 0\left(\overline{\varpi_{3}} \geq 0\right.$ can be demonstrated in a similar fashion) by noticing that if $\underline{\varepsilon}^{(i)} \geq 0$ and $\bar{\varepsilon}^{(i)} \geq 0$, the $i$-th terms in (50) equal the $i$-th terms in (28), such that nonnegativity is assured as long as ${\underline{x_{R}}}^{(i)} \leq x_{R}^{(i)} \leq{\overline{x_{R}}}^{(i)}$. This is necessarily true, since from (2), it follows that:

$$
\begin{aligned}
& x_{R}-\underline{x_{R}}=R(\vartheta)(y-\underline{x}-v) \\
& \overline{x_{R}}-x_{R}=R(\vartheta)(\bar{x}-y+v)
\end{aligned}
$$

Applying the results in [26] and taking into account that $|v| \leq V$ (see Assumption 1), it follows that $-|R(\vartheta)| V \leq v \leq|R(\vartheta)| V$, which leads to:

$$
\begin{aligned}
& x_{R}-\underline{x_{R}} \in[\underline{\varepsilon}, R(\vartheta)(y-\underline{x})+|R(\vartheta)| V] \\
& \overline{x_{R}}-x_{R} \in[\bar{\varepsilon}, R(\vartheta)(\bar{x}-y)+|R(\vartheta)| V]
\end{aligned}
$$

Hence, $x_{R}^{(i)}-\underline{x}_{R}^{(i)} \geq \underline{\varepsilon}^{(i)} \geq 0$ and ${\overline{x_{R}}}^{(i)}-x_{R}^{(i)} \geq \bar{\varepsilon}^{(i)} \geq 0$, which assures nonnegativity of the $i$-th terms in (50) for $\underline{\varepsilon}^{(i)} \geq 0$ and $\bar{\varepsilon}^{(i)} \geq 0$.

Let us consider the case when $\underline{\varepsilon}^{(i)}<0$ (the case when $\bar{\varepsilon}^{(i)}<0$ follows a similar reasoning, thus it is omitted). When $\underline{\varepsilon}^{(i)}<0$, the $i$-th terms in (50) can be expressed as:

$$
\Delta A_{R}^{(i)}(\vartheta) x_{R}^{(i)}+\overline{\Delta A_{R}^{(i)}}(\vartheta) x_{R}^{(i)}{ }^{-}-\underline{\Delta A_{R}^{(i)}}(\vartheta){\overline{x_{R}}}^{(i)^{+}}
$$

which are positive if $x_{R}^{(i)} \leq x_{R}^{(i)} \leq{\overline{x_{R}}}^{(i)}$. It is straightforward that $x_{R}^{(i)} \leq{\overline{x_{R}}}^{(i)}$ due to $\bar{\varepsilon}^{(i)} \geq 0$. On the other hand, from (2), it follows that:

$$
x_{R}=R(\vartheta)(y-v)
$$

that, taking into account that $-|R(\vartheta)| V \leq v \leq|R(\vartheta)| V$, leads to:

$$
x_{R} \in\left[\underline{\sim}_{R}, \tilde{x}_{R}\right]
$$

which proves that $x_{R}^{(i)} \geq x_{R}^{(i)}$, so that $\underline{\varpi_{3}}$ (similarly, $\overline{\varpi_{3}}$ ) is nonnegative. Also, the nonnegativity of $\underline{\varpi_{5}}$, $\overline{\omega_{5}}$ follows directly from Assumption 2 and the results in [26]. Then, since $\underline{F}(\vartheta), \bar{F}(\vartheta) \in \mathbb{M}^{n_{x} \times n_{x}}$, any solution of (24)-(25) with $v_{u n}=0$ is elementwise nonnegative for all $t \geq 0$.

Let us show that the variables $\underline{x_{R}}$ and $\overline{x_{R}}$ stay bounded $\forall t \geq 0$. Without loss of generality, let us consider the case where:

$$
\left\{\begin{array} { l l } 
{ \underline { \varepsilon } ^ { ( i ) } < 0 } & { i \in \mathscr { N } _ { 1 } } \\
{ \underline { \varepsilon } ^ { ( i ) } \geq 0 } & { i \in \mathscr { S } _ { 1 } }
\end{array} \quad \left\{\begin{array}{ll}
\bar{\varepsilon}^{(i)}<0 & i \in \mathscr{N}_{2} \\
\bar{\varepsilon}^{(i)} \geq 0 & i \in \mathscr{S}_{2}
\end{array}\right.\right.
$$

with $\mathscr{N}_{1} \cap \mathscr{S}_{1}=\emptyset, \mathscr{N}_{2} \cap \mathscr{S}_{2}=\emptyset$ and $\mathscr{N}_{1} \cup \mathscr{S}_{1}=\mathscr{N}_{2} \cup \mathscr{S}_{2}=\left\{1, \ldots, n_{x}\right\}$. In this case, the equations that rule the dynamics of $\underline{x_{R}}$ and $\overline{x_{R}}$ can be written as:

$$
\begin{aligned}
& \dot{\dot{x_{R}}}=\underline{F}(\vartheta) \underline{x_{R}}+\underline{g}\left(\underline{x_{R}}, \overline{x_{R}}\right)+\underline{\delta} \\
& \dot{\overline{x_{R}}}=\left(\bar{F}(\vartheta)+\overline{\Delta A_{R}^{\mathscr{S}_{2}}}(\vartheta)\right) \overline{x_{R}}+\bar{g}\left(\underline{x_{R}}, \overline{x_{R}}\right)+\bar{\delta}
\end{aligned}
$$


where (similar expressions hold for $\bar{g}\left(\underline{x_{R}}, \overline{x_{R}}\right)$ and $\left.\bar{\delta}\right)$ :

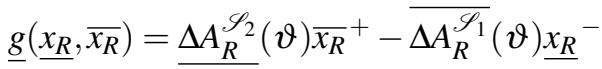

$$
\begin{aligned}
& \underline{\delta}=R(\vartheta) \underline{T}(\vartheta) \underline{K}(\vartheta) y-|R(\vartheta) \underline{T}(\vartheta) \underline{K}(\vartheta)| V+R(\vartheta) B(\vartheta) v+R(\vartheta) k(\vartheta)+\underline{d_{R}}(\vartheta) \\
& +\Delta A_{R}^{\left(\mathscr{N}_{2}\right)}(\vartheta) \tilde{x}_{R}{ }^{+}-\overline{\Delta A_{R}^{\left(\mathscr{N}_{1}\right)}}(\vartheta) \underline{x}_{R}{ }^{-}+\underline{\Delta B_{R}}(\vartheta) v^{+}-\overline{\Delta B_{R}}(\vartheta) v^{-} \\
& +\underline{\Delta B_{u n, R}}(\vartheta) \overline{v_{u n}}+-\overline{\Delta B_{u n, R}}(\vartheta) \underline{v_{u n}}{ }^{-}
\end{aligned}
$$

Also in this case, similarly to the proof of Theorem 1 , for all $\vartheta \in \Theta$ :

$$
\left|\phi_{g}(\xi)\right| \leq \eta\left(\vartheta, \mathscr{S}_{1}, \mathscr{S}_{2}\right)|\xi|
$$

with $\eta\left(\vartheta, \mathscr{S}_{1}, \mathscr{S}_{2}\right)$ given by (46), while the inputs $\underline{\delta}$ and $\bar{\delta}$ are bounded because of Assumptions $1-2$, and the fact that $x \in \mathscr{L}_{\infty}^{n_{x}}, v \in \mathscr{L}_{\infty}^{n_{v}}, k \in \mathscr{L}_{\infty}^{n_{x}}$ and $|v| \leq V$. Hence, it can be shown through a Lyapunov function $V=\xi^{T} P \xi$ and input to state reasoning [42] that if (45) holds, then $\underline{x_{R}}, \overline{x_{R}} \in \mathscr{L}_{\infty}^{n_{x}}$. Since the indices contained in the sets $\mathscr{S}_{1}$ and $\mathscr{S}_{2}$ are not known a priori, it follows that (45) should hold $\forall \mathscr{S}_{1}, \mathscr{S}_{2} \in \mathscr{P}\left(\left\{1, \ldots, n_{x}\right\}\right)$ in order to guarantee the boundedness of $\underline{x_{R}}$ and $\overline{x_{R}}$, thus completing the proof.

At this point, using Lemma 1, the following theorem provides the conditions which should be satisfied by the gains $\underline{K}(\vartheta), \bar{K}(\vartheta), \underline{T}(\vartheta)$ and $\bar{T}(\vartheta)$ in order to solve Problem 2 .

\section{Theorem 2}

Given the matrix $S \in \mathbb{R}^{n_{x} \times n_{v_{u n}}}$, let Assumptions 1-2 be satisfied, $x \in \mathscr{L}_{\infty}^{n_{x}}, v \in \mathscr{L}_{\infty}^{n_{v}}, k \in \mathscr{L}_{\infty}^{n_{x}}$, the matrix function $R(\vartheta)$ be such that (36) holds, and the proportional integral interval unknown input observer be given by (13)-(14), (16)-(17) and (41)-(44). Then, if there exist matrix functions $\underline{\Gamma}(\vartheta), \bar{\Gamma}(\vartheta) \in \mathbb{D}^{n} v_{u n} \times n_{v_{u n}}$ and matrix functions $\underline{S}^{*}(\vartheta), \bar{S}^{*}(\vartheta) \in \mathbb{R}^{n_{x} \times n_{x}}$ such that $\underline{F}(\vartheta), \bar{F}(\vartheta)$, defined as in (19)-(20), with:

$$
\begin{aligned}
\underline{T}(\vartheta) \underline{K}(\vartheta)= & {\left[A(\vartheta) R(\vartheta)^{-1} S+R(\vartheta)^{-1} \dot{R}(\vartheta) R(\vartheta)^{-1} S-R(\vartheta)^{-1} S \underline{\Gamma}(\vartheta)\right] B_{u n}(\vartheta)^{\dagger} } \\
& +\underline{S}^{*}(\vartheta)\left[I-B_{u n}(\vartheta) B_{u n}(\vartheta)^{\dagger}\right] \\
\bar{T}(\vartheta) \bar{K}(\vartheta)= & {\left[A(\vartheta) R(\vartheta)^{-1} S+R(\vartheta)^{-1} \dot{R}(\vartheta) R(\vartheta)^{-1} S-R(\vartheta)^{-1} S \bar{\Gamma}(\vartheta)\right] B_{u n}(\vartheta)^{\dagger} } \\
& +\bar{S}^{*}(\vartheta)\left[I-B_{u n}(\vartheta) B_{u n}(\vartheta)^{\dagger}\right]
\end{aligned}
$$

are Metzler, then the relations (37)-(38) are satisfied provided that (11) holds. Moreover, if (12) holds, then also (10) is satisfied.

In addition, if there exist $P \in \mathbb{S}^{2 n_{x} \times 2 n_{x}}, P \succ 0, Q \in \mathbb{S}^{2 n_{x} \times 2 n_{x}}, Q \succ 0$ and constants $\varepsilon_{1}, \varepsilon_{2}, \gamma>0$ such that (45), with $\eta\left(\vartheta, \mathscr{S}_{1}, \mathscr{S}_{2}\right)$ and $G\left(\vartheta, \mathscr{S}_{2}\right)$ defined as in (46)-(47), is verified $\forall \mathscr{S}_{1}, \mathscr{S}_{2} \in$ $\mathscr{P}\left(\left\{1, \ldots, n_{x}\right\}\right)$, then $\underline{x_{R}}, \overline{x_{R}} \in \mathscr{L}_{\infty}^{n_{x}}$.

Proof of Theorem 2: As shown previously, by using the unknown input interval observer (13)(14), (16)-(17) and (41)-(44), the dynamics of the interval estimation errors $\underline{e}, \bar{e}$ follow (48)-(49). 
Looking at (36), it is straightforward that for guaranteeing (10) and (37)-(38), in addition to the conditions of Lemma 1, the columns of $S$ need to correspond to eigenvectors of the matrices $\underline{F}(\vartheta)$ and $\bar{F}(\vartheta)$, i.e.:

$$
\begin{aligned}
& \underline{F}(\vartheta) S=S \underline{\Gamma}(\vartheta) \\
& \bar{F}(\vartheta) S=S \bar{\Gamma}(\vartheta)
\end{aligned}
$$

where $\underline{\Gamma}(\vartheta), \bar{\Gamma}(\vartheta) \in \mathbb{R}^{n v_{u n} \times n_{v_{u n}}}$ contain some of the eigenvalues of $\underline{F}(\vartheta), \bar{F}(\vartheta)$ (the ones that correspond to the eigenvectors that are columns of $S$ ).

Taking into account (36) and (19)-(20), it is easy to see that (54)-(55) are equivalent to:

$$
\begin{aligned}
& \underline{T}(\vartheta) \underline{K}(\vartheta) B_{u n}(\vartheta)=A(\vartheta) R(\vartheta)^{-1} S+R(\vartheta)^{-1} \dot{R}(\vartheta) R(\vartheta)^{-1} S-R(\vartheta)^{-1} S \underline{\Gamma}(\vartheta) \\
& \bar{T}(\vartheta) \bar{K}(\vartheta) B_{u n}(\vartheta)=A(\vartheta) R(\vartheta)^{-1} S+R(\vartheta)^{-1} \dot{R}(\vartheta) R(\vartheta)^{-1} S-R(\vartheta)^{-1} S \bar{\Gamma}(\vartheta)
\end{aligned}
$$

whose solutions can be expressed as (52)-(53), which completes the proof.

\subsection{Design conditions}

Also in this case, it is possible to derive conditions for performing the design, as specified by the following corollary.

\section{Corollary 2}

Given the matrix $S \in \mathbb{R}^{n_{x} \times n_{v_{u n}}}$, let Assumptions 1-2 be satisfied, $x \in \mathscr{L}_{\infty}^{n_{x}}, v \in \mathscr{L}_{\infty}^{n_{v}}, k \in \mathscr{L}_{\infty}^{n_{x}}$ and the matrix function $R(\vartheta)$ be such that (36) holds. Also, let us assume that there exist an elementwise nonnegative block-diagonal matrix $P$ as in (30), with $\underline{P}, \bar{P} \in \mathbb{S}^{n_{x} \times n_{x}}, \underline{P}, \bar{P} \succ 0$, a matrix function:

$$
W_{S}(\vartheta)=\left(\begin{array}{cc}
\underline{W}_{S}(\vartheta) & 0 \\
0 & \bar{W}_{S}(\vartheta)
\end{array}\right)
$$

with $\underline{W_{S}}(\vartheta), \overline{W_{S}}(\vartheta) \in \mathbb{R}^{n_{x} \times n_{x}}$, a matrix $Q \in \mathbb{S}^{2 n_{x} \times 2 n_{x}}$, a sufficiently large matrix function $\Sigma \in \mathbb{D}_{+}^{2 n_{x} \times 2 n_{x}}$ and constants $\varepsilon_{1}, \varepsilon_{2}, \gamma>0$ such that:

$$
\begin{gathered}
\left(\begin{array}{cc}
H e\left\{P \Xi\left(\vartheta, \mathscr{S}_{2}\right)+W_{S}(\vartheta) \Upsilon^{*}(\vartheta)\right\}+\left(\varepsilon_{1}+\varepsilon_{2}\right) P+Q+\gamma \eta\left(\vartheta, \mathscr{S}_{1}, \mathscr{S}_{2}\right)^{2} I_{2 n_{x}} & 0 \\
0 & \varepsilon_{1}^{-1} P-\gamma I_{2 n_{x}}
\end{array}\right) \preceq 0 \\
P\left(\begin{array}{cc}
\Xi(\vartheta) & 0 \\
0 & \bar{\Xi}\left(\vartheta, \mathscr{S}_{2}\right)-\overline{\Delta A_{R}^{\mathscr{S}}(\vartheta)}
\end{array}\right)-W_{S}(\vartheta) \Upsilon^{*}(\vartheta)+P \Sigma(\vartheta) \geq 0
\end{gathered}
$$

with $\eta\left(\vartheta, \mathscr{S}_{1}, \mathscr{S}_{2}\right)$ defined as in (46) and:

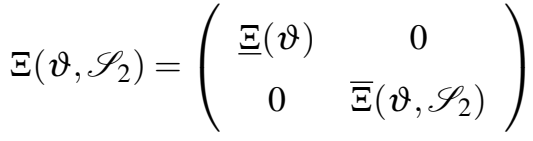

$$
\begin{aligned}
& \Upsilon^{*}(\vartheta)=\left(\begin{array}{cc}
\left(I-B_{u n}(\vartheta) B_{u n}(\vartheta)^{\dagger}\right) R(\vartheta)^{-1} & 0 \\
0 & \left(I-B_{u n}(\vartheta) B_{u n}(\vartheta)^{\dagger}\right) R(\vartheta)^{-1}
\end{array}\right)
\end{aligned}
$$




$$
\begin{aligned}
\underline{\Xi}(\vartheta)= & R(\vartheta) A(\vartheta) R(\vartheta)^{-1}\left(I-S B_{u n}(\vartheta)^{\dagger} R(\vartheta)^{-1}\right)+\dot{R}(\vartheta) R(\vartheta)^{-1} \\
& +\left(S \underline{\Gamma}(\vartheta)-\dot{R}(\vartheta) R(\vartheta)^{-1} S\right) B_{\text {un }}(\vartheta)^{\dagger} R(\vartheta)^{-1} \\
\bar{\Xi}\left(\vartheta, \mathscr{S}_{2}\right) & =R(\vartheta) A(\vartheta) R(\vartheta)^{-1}\left(I-S B_{u n}(\vartheta)^{\dagger} R(\vartheta)^{-1}\right)+\dot{R}(\vartheta) R(\vartheta)^{-1} \\
& +\left(S \bar{\Gamma}(\vartheta)-\dot{R}(\vartheta) R(\vartheta)^{-1} S\right) B_{u n}(\vartheta)^{\dagger} R(\vartheta)^{-1}+\overline{\Delta A_{R}^{\mathscr{S}}(\vartheta)}
\end{aligned}
$$

Then, the proportional integral interval unknown input observer (13)-(14), (16)-(17) and (41)-(44) with matrices satisfying (52)-(53), with:

$$
\left(\begin{array}{cc}
\underline{S}^{*}(\vartheta) & 0 \\
0 & \bar{S}^{*}(\vartheta)
\end{array}\right)=\left(\begin{array}{cc}
\underline{P} R(\vartheta) & 0 \\
0 & \bar{P} R(\vartheta)
\end{array}\right)^{-1} W_{S}(\vartheta)
$$

is such that the relations (37)-(38) are satisfied provided that (11) holds. Moreover, if (12) holds, then also (10) is satisfied, with $\underline{x_{R}}, \overline{x_{R}} \in \mathscr{L}_{\infty}^{n_{x}}$.

Proof of Corollary 2: (56) can be obtained from (45) through the change of variables:

$$
W_{S}(\vartheta)=\left(\begin{array}{cc}
\underline{P} R(\vartheta) \underline{S}^{*}(\vartheta) & 0 \\
0 & \bar{P} R(\vartheta) \bar{S}^{*}(\vartheta)
\end{array}\right)
$$

which explains why $\underline{S}^{*}(\vartheta)$ and $\bar{S}^{*}(\vartheta)$ are calculated as in (58). On the other hand, (57) corresponds to the verification of the Metzler property [25].

Also in this case, the infinite number of conditions given by Theorem 2 and Corollary 2 can be brought to a finite number by gridding the varying parameter space $\Theta$ using $N$ points $\vartheta_{i}, i=1, \ldots, N$. The details are skipped for the sake of brevity.

\section{APPLICATION TO THE UAV}

\subsection{Nonlinear model}

The longitudinal equations of a UAV, under normal flight conditions (low angle-of-attack) consist of two equations for the airspeed components $(u$ and $w$, i.e. the horizontal and the vertical components, 
respectively), an equation for the pitch rate $q$ and an equation for the pitch angle $\theta$ [49]:

$$
\begin{aligned}
\dot{u}= & -q w-g \sin \theta+\frac{\rho V_{a}^{2} S}{2 m}\left[-\left(C_{D_{0}}+C_{D_{\alpha}} \alpha\right) \cos \alpha+\left(C_{L_{0}}+C_{L_{\alpha}} \alpha\right) \sin \alpha\right. \\
& \left.+\left(C_{L_{q}} \sin \alpha-C_{D_{q}} \cos \alpha\right) \frac{c q}{2 V_{a}}+\left(C_{L_{\delta_{e}}} \sin \alpha-C_{D_{\delta_{e}}} \cos \alpha\right) \delta_{e}\right]+\frac{\rho S_{p r o p} C_{p r o p}}{2 m}\left(k_{m}^{2} \delta_{t}^{2}-V_{a}^{2}\right) \\
\dot{w}= & q u+g \cos \theta+\frac{\rho V_{a}^{2} S}{2 m}\left[-\left(C_{D_{0}}+C_{D_{\alpha}} \alpha\right) \sin \alpha-\left(C_{L_{0}}+C_{L_{\alpha}} \alpha\right) \cos \alpha\right. \\
& \left.-\left(C_{D_{q}} \sin \alpha+C_{L_{q}} \cos \alpha\right) \frac{c q}{2 V_{a}}-\left(C_{D_{\delta_{e}}} \sin \alpha+C_{L_{\delta_{e}}} \cos \alpha\right) \delta_{e}\right] \\
\dot{q}= & \frac{\rho V_{a}^{2} S c}{2 J_{y}}\left(C_{m_{0}}+C_{m_{\alpha}} \alpha+C_{m_{q}} \frac{c q}{2 V_{a}}+C_{m_{\delta_{e}}} \delta_{e}\right) \\
\dot{\theta}= & q
\end{aligned}
$$

where $\rho$ is the air density, $S$ is the wing surface area, $m$ is the airframe mass, $\alpha$ is the angle-ofattack, $c$ is the mean aerodynamic chord of the wing, $S_{\text {prop }}$ is the area of the propeller, $k_{m}$ is the constant that specifies the efficiency of the motor, $J_{y}$ is an element of the inertia matrix and $V_{a}$ is the total airspeed with respect to the air mass. The inputs entering the system are the thrust command $\delta_{t}$ and the elevator deflection $\delta_{e}$. Finally, the non-dimensional coefficients $C_{i}$ are usually referred to as stability and control derivatives. Even without any icing or other faults, it is assumed that the stability and control derivatives are uncertain, i.e. they can be expressed as:

$$
C_{i}=\bar{C}_{i}+\Delta C_{i}
$$

where $\bar{C}_{i}$ is the nominal value, which is assumed to be known, and $\Delta C_{i}$ corresponds to the uncertainty, which is unknown but bounded by known bounds. In the following, a Zagi Flying Wing UAV is used as case study, with the parameters listed in Table I [49]. Assuming that the UAV is equipped with measurement devices such as pitot tubes, GPS and inertial sensors [49] and estimators [50], all state variables are supposed to be available and hence the output equation reads as (2).

Table I. System nominal parameters values

\begin{tabular}{cccccc}
\hline Param. & Value & Param. & Value & Param. & Value \\
\hline$m$ & $1.56 \mathrm{~kg}$ & $\bar{C}_{L_{0}}$ & 0.09167 & $\bar{C}_{D_{q}}$ & 0 \\
$J_{y}$ & $0.0576 \mathrm{~kg} \mathrm{~m}^{2}$ & $\bar{C}_{D_{0}}$ & 0.01631 & $\bar{C}_{m_{q}}$ & -1.3990 \\
$S$ & $0.2589 \mathrm{~m}^{2}$ & $\bar{C}_{m_{0}}$ & -0.02338 & $\bar{C}_{L_{\delta_{e}}}$ & 0.2724 \\
$c$ & $0.3302 m$ & $\bar{C}_{L_{\alpha}}$ & 3.5016 & $\bar{C}_{D_{\delta_{e}}}$ & 0.3045 \\
$S_{\text {prop }}$ & $0.0314 \mathrm{~m}^{2}$ & $\bar{C}_{D_{\alpha}}$ & 0.2108 & $\bar{C}_{m_{\delta_{e}}}$ & -0.3254 \\
$\rho$ & $1.2682 \mathrm{~kg} / \mathrm{m}^{3}$ & $\bar{C}_{m_{\alpha}}$ & -0.5675 & $C_{\text {prop }}$ & 1.0 \\
$k_{m}$ & 20 & $\bar{C}_{L_{q}}$ & 2.8932 & & \\
\hline \multicolumn{7}{c}{} & & & &
\end{tabular}


The wind acceleration acts as an additive disturbance vector $\mathscr{W}$ given by:

$$
\mathscr{W}=\left(\begin{array}{cc}
-\cos \theta & -\sin \theta \\
-\sin \theta & \cos \theta \\
0 & 0 \\
0 & 0
\end{array}\right)\left(\begin{array}{c}
\dot{\omega}_{x} \\
\dot{\omega}_{z}
\end{array}\right)=H_{1}(\theta) \dot{\omega}_{x}+H_{2}(\theta) \dot{\omega}_{z}
$$

where $\dot{\omega}_{x}$ and $\dot{\omega}_{z}$ are the wind accelerations in the horizontal and vertical directions in the inertial frame, respectively.

In this paper, we will consider multiplicative actuator faults, described by actuator effectiveness terms ranging between two extreme values, i.e. 0 (total loss) and 1 (healthy behaviour). These faults can be represented as an unknown input term $\mathscr{F}$ given by:

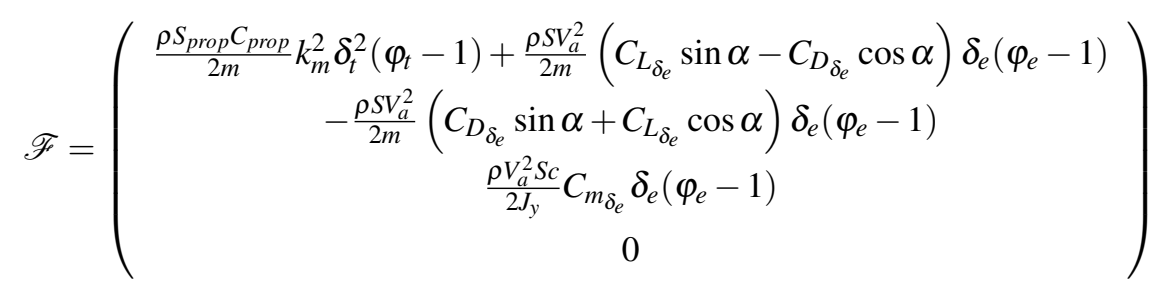

where $\varphi_{t}$ and $\varphi_{e}$ represent the effectiveness of propulsion and elevator, respectively.

\subsection{Quasi-LPV model}

Using the nonlinear embedding in the parameters approach [51,52] and taking into account that $V_{a}=\sqrt{u^{2}+w^{2}}$ and $\alpha=\arctan \left(\frac{w}{u}\right)$, the nonlinear model (60)-(63) can be brought to a quasi-LPV form [53, 54] as in (1), with $x=(u, w, q, \theta)^{T}, v=\left(\delta_{t}^{2}, \delta_{e}\right)^{T}, v_{u n}=\left(\left(\varphi_{t}-1\right) \delta_{t}^{2},\left(\varphi_{e}-1\right) \delta_{e}, \dot{\omega}_{z}\right)^{T}$, $k(\theta)=(-g \sin \theta, g \cos \theta, 0,0)^{T}$ and $d(\theta)=\left(-\dot{\omega}_{x} \cos \theta,-\dot{\omega}_{x} \sin \theta, 0,0\right)^{T}$. The matrix functions $A(\cdot)$, $B(\cdot), B_{u n}(\cdot), \Delta A(\cdot), \Delta B(\cdot), \Delta B_{u n}(\cdot)$ have the following structure (the expressions of the coefficients appearing in the matrices are reported in the Appendix):

$$
\begin{aligned}
& A(\cdot)=\left(\begin{array}{cccc}
\bar{a}_{11}(\cdot) & \bar{a}_{12}(\cdot) & \bar{a}_{13}(\cdot) & 0 \\
\bar{a}_{21}(\cdot) & \bar{a}_{22}(\cdot) & \bar{a}_{23}(\cdot) & 0 \\
\bar{a}_{31}(\cdot) & \bar{a}_{32}(\cdot) & \bar{a}_{33}(\cdot) & 0 \\
0 & 0 & 1 & 0
\end{array}\right) \quad \Delta A(\cdot)=\left(\begin{array}{cccc}
\Delta a_{11}(\cdot) & \Delta a_{12}(\cdot) & \Delta a_{13}(\cdot) & 0 \\
\Delta a_{21}(\cdot) & \Delta a_{22}(\cdot) & \Delta a_{23}(\cdot) & 0 \\
\Delta a_{31}(\cdot) & \Delta a_{32}(\cdot) & \Delta a_{33}(\cdot) & 0 \\
0 & 0 & 0 & 0
\end{array}\right) \\
& B(\cdot)=\left(\begin{array}{cc}
b_{11} & \bar{b}_{12}(\cdot) \\
0 & \bar{b}_{22}(\cdot) \\
0 & \bar{b}_{32}(\cdot) \\
0 & 0
\end{array}\right) \quad \Delta B(\cdot)=\left(\begin{array}{cc}
0 & \Delta b_{12}(\cdot) \\
0 & \Delta b_{22}(\cdot) \\
0 & \Delta b_{32}(\cdot) \\
0 & 0
\end{array}\right) \\
& B_{u n}(\cdot)=\left(\begin{array}{ccc}
b_{11} & \bar{b}_{12}(\cdot) & -\sin \theta \\
0 & \bar{b}_{22}(\cdot) & \cos \theta \\
0 & \bar{b}_{32}(\cdot) & 0 \\
0 & 0 & 0
\end{array}\right) \quad \Delta B_{u n}(\cdot)=\left(\begin{array}{ccc}
0 & \Delta b_{12}(\cdot) & 0 \\
0 & \Delta b_{22}(\cdot) & 0 \\
0 & \Delta b_{32}(\cdot) & 0 \\
0 & 0 & 0
\end{array}\right)
\end{aligned}
$$


Remark 9: The nonlinear embedding in the parameters approach [51, 52] produces an exact quasi-LPV representation of a nonlinear system, which means that no approximation is involved, and the obtained model represents the system's behavior (in this case, the UAV's) over the whole operating region of the state space. This is in contrast with other methods which, being based on linearization, produce LPV models that are closer to the LTI ones obtained under trim conditions, see e.g. [55]. As discussed by [56], conventional approaches to generate an LPV model based on Jacobian linearizations at trim points fail in representing the dynamics at non-trim conditions. Conversely, when the nonlinear terms are substituted for other functions in quasi-LPV form (as in the nonlinear embedding approach), the obtained model can be applied for both trim and non-trim conditions.

Remark 10: The coefficients reported in the Appendix do not take into account the inaccuracy about the value of the scheduling variables due to the measurement noise $v$. It is possible to consider coefficients that take into account the measurement noise in order to increase the robustness of the designed interval observer. However, it is worth highlighting the fact that the measurement noise is typically a high-frequency zero-mean signal, so its effect on the interval observer's estimation due to inaccuracies in the scheduling variables is smoothed out by the system's dynamics, which behave as a low-pass filter. On the other hand, the parametric uncertainties are constant deviations from the nominal values, which cause dangerous low-frequency biases in the observer's estimation. For this reason, it is of paramount importance to consider them in the interval estimation.

\subsection{Icing effects}

The accretion of ice on the UAV surfaces modifies the stability and control derivatives according to the following linear model [57]:

$$
C_{i}^{*}=\left(1+\eta K_{i}\right) C_{i}
$$

where $\eta$ is the icing severity factor and the coefficient $K_{i}$ depends on the UAV design and atmospheric conditions. The clean condition corresponds to $\eta=0$, while the all iced condition occurs for $\eta=\eta_{\max }[7]$.

As a consequence, the overall icing effect can be modeled as an additive time-dependent disturbance term $\mathscr{E}(u, w, q) \eta$, where $\eta$ is a scalar unknown quantity and the vector $\mathscr{E}(u, w, q)$ is given by:

$$
\mathscr{E}(u, w, q)=\left(\begin{array}{llll}
\mathscr{E}_{1}(u, w, q) & \mathscr{E}_{2}(u, w, q) & \mathscr{E}_{3}(u, w, q) & 0
\end{array}\right)^{T}
$$


with:

$$
\begin{aligned}
\mathscr{E}_{1}(u, w, q) & =\frac{\rho V_{a}^{2} S}{2 m}\left[\left(K_{L_{0}} C_{L_{0}}+K_{L_{\alpha}} C_{L_{\alpha}} \alpha\right) \sin \alpha-\left(K_{D_{0}} C_{D_{0}}+K_{D_{\alpha}} C_{D_{\alpha}} \alpha\right) \cos \alpha\right. \\
& \left.+\left(K_{L_{q}} C_{L_{q}} \sin \alpha-K_{D_{q}} C_{D_{q}} \cos \alpha\right) \frac{c q}{2 V_{a}}+\left(K_{L_{\delta_{e}}} C_{L_{\delta_{e}}} \sin \alpha-K_{D_{\delta_{e}}} C_{D_{\delta_{e}}} \cos \alpha\right) \delta_{e}\right] \\
\mathscr{E}_{2}(u, w, q) & =-\frac{\rho V_{a}^{2} S}{2 m}\left[\left(K_{D_{0}} C_{D_{0}}+K_{D_{\alpha}} C_{D_{\alpha}} \alpha\right) \sin \alpha+\left(K_{L_{0}} C_{L_{0}}+K_{L_{\alpha}} C_{L_{\alpha}} \alpha\right) \cos \alpha\right. \\
& \left.+\left(K_{D_{q}} C_{D_{q}} \sin \alpha+K_{L_{q}} C_{L_{q}} \cos \alpha\right) \frac{c q}{2 V_{a}}+\left(K_{D_{\delta_{e}}} C_{D_{\delta_{e}}} \sin \alpha+K_{L_{\delta_{e}}} C_{L_{\delta_{e}}} \cos \alpha\right) \delta_{e}\right] \\
\mathscr{E}_{3}(u, w, q) & =\frac{\rho V_{a}^{2} S c}{2 J_{y}}\left(K_{m_{0}} C_{m_{0}}+K_{m_{\alpha}} C_{m_{\alpha}} \alpha+K_{m_{q}} C_{m_{q}} \frac{c q}{2 V_{a}}+K_{m_{\delta_{e}}} C_{m_{\delta_{e}}} \delta_{e}\right)
\end{aligned}
$$

The icing severity factor evolves according to the law:

$$
\eta=\mathscr{N}(\varpi) \chi
$$

where $\mathscr{N}(\cdot)$ is a nonlinear function, $\varpi$ is the fraction of water freezing at a point on a surface with respect to the water impinging on the surface, and $\chi$ is the accumulation parameter. It has been observed experimentally that the icing severity factor achieves its maximum $\eta_{\max }$ when the freezing fraction $\varpi$ is close to the value $\varpi_{g}=0.2$, while it decreases to a steady value as $\varpi$ approaches 1 .

It is worth noting that icing may likely also alter the airspeed measurements, as the pitot tube may be clogged by the ice, usually leading to an over-estimation of the airspeed caused by the increased pressure. However, in this work, it is assumed that the pitot tube is equipped with heating devices, which allow a straightforward accommodation of icing effects on sensors.

\subsection{Fault/icing diagnosis}

Let us notice that, as long as $\cos \theta \neq 0$, the following condition holds:

$$
\mathscr{E}(u, w, q)=\mathscr{E}(t) \in \operatorname{span}\left[\begin{array}{lll}
B_{1} & B_{2}(u, w) & H_{2}(\theta)
\end{array}\right] \forall t \geq 0
$$

Since the actuator effectiveness ranges between 0 and 1, it is straightforward that (34) is satisfied with $\underline{v_{u n}}=\left(-\delta_{t}^{2}, \min \left(0,-\delta_{e}\right),-\dot{\omega}_{z}^{\max }\right)^{T}$ and $\overline{v_{u n}}=\left(0, \max \left(0,-\delta_{e}\right), \dot{\omega}_{z}^{\max }\right)^{T}$, where $\dot{\omega}_{z}^{\max }$ is the maximum value for $\left|\dot{\omega}_{z}\right|$. Due to the actuator actions and the wind acceleration being limited in magnitude, it follows that $v_{u n}, \overline{v_{u n}} \in \mathscr{L}_{\infty}^{n_{v_{u n}}}, d \in \mathscr{L}_{\infty}^{n_{x}}$, and it is reasonable that there exists a known bound $V$ on the noise. Hence, Assumptions 1-2 are satisfied and the robust fault/icing diagnosis can be achieved using the LPV proportional integral interval unknown observer given by (13)-(14), (16)-(17) and (41)-(44). 
Then, by recalling the definition of Problem 2, it is possible to choose the matrix $S$ as ${ }^{\ddagger}$ :

$$
S=\left(\begin{array}{lll}
1 & 0 & 0 \\
0 & 1 & 0 \\
0 & 0 & 1 \\
0 & 0 & 0
\end{array}\right)
$$

which, under single fault assumption, leads to the following fault/icing diagnosis algorithm, based on conditions (37)-(38):

\section{Diagnosis Algorithm.}

$$
\begin{aligned}
& \text { if }\left\{\begin{array}{lll}
\underline{\varepsilon}^{(1)} \geq 0 & A N D \quad \bar{\varepsilon}^{(1)} \geq 0 \\
\underline{\varepsilon}^{(2)} \geq 0 & A N D \quad \bar{\varepsilon}^{(2)} \geq 0 \\
\underline{\varepsilon}^{(3)} \geq 0 & A N D \quad & \bar{\varepsilon}^{(3)} \geq 0
\end{array} \quad\right. \text { then " no faults/no icing", }
\end{aligned}
$$

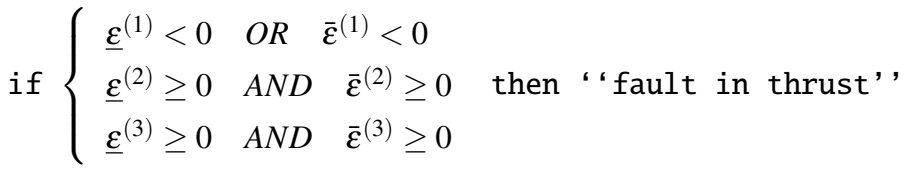

$$
\begin{aligned}
& \text { if }\left\{\begin{array}{l}
\underline{\varepsilon}^{(1)} \geq 0 \quad A N D \quad \bar{\varepsilon}^{(1)} \geq 0 \\
\underline{\varepsilon}^{(2)}<0 \quad \text { OR } \quad \bar{\varepsilon}^{(2)}<0 \\
\underline{\varepsilon}^{(3)} \geq 0 \quad A N D \quad \bar{\varepsilon}^{(3)} \geq 0
\end{array} \quad\right. \text { then " "fault in elevator", }
\end{aligned}
$$

In other words, as long as all the signals $\underline{\varepsilon}^{(i)}, \bar{\varepsilon}^{(i)}$ are nonnegative, the behavior of the UAV is compatible with the considered sources of uncertainty. When either $\underline{\varepsilon}^{(1)}$ or $\bar{\varepsilon}^{(1)}\left(\underline{\varepsilon}^{(2)}\right.$ or $\left.\bar{\varepsilon}^{(2)}\right)$ is the only signal to become negative, a fault in thrust (in elevator) can be indicated. On the other hand, if multiple signals become negative, then the diagnoser indicates that icing has occurred.

By imposing condition (36), and taking into account the structure of $B_{u n}(\vartheta)$ in (66), it is possible to calculate the matrix $R(\vartheta)$, as follows:

$$
R(\vartheta)=\left(\begin{array}{cccc}
1 / b_{11} & \tan \theta / b_{11} & r_{13}(\vartheta) & 0 \\
0 & 0 & 1 / \bar{b}_{32}(\vartheta) & 0 \\
0 & 1 / \cos \theta & r_{33}(\vartheta) & 0 \\
0 & 0 & 0 & r_{44}(\vartheta)
\end{array}\right)
$$

with:

$$
\begin{aligned}
& r_{13}(\vartheta)=-\frac{1}{\bar{b}_{32}(\vartheta)}\left[\frac{\bar{b}_{12}(\vartheta)}{b_{11}}+\frac{\sin \theta}{\cos \theta} \frac{\bar{b}_{22}(\vartheta)}{b_{11}}\right] \\
& r_{33}(\vartheta)=-\frac{\bar{b}_{22}(\vartheta)}{\bar{b}_{32}(\vartheta) \cos \theta}
\end{aligned}
$$

\footnotetext{
${ }^{\ddagger}$ Infinite choices of $S$ are possible, but they would lead to more complicated diagnosis algorithms.
} 
and an arbitrary $r_{44}(\vartheta) \neq 0$ (in the following, it is chosen as $r_{44}(\vartheta)=1$ ).

Then, the lower and upper bounds for $d_{R}(\theta)=R(\vartheta) d(\theta)=$ $\left(-\dot{\omega}_{x}(\cos \theta+\sin \theta \tan \theta) / b_{11}, 0,-\dot{\omega}_{x} \tan \theta, 0\right)^{T}$ can be calculated as $\overline{d_{R}}(\theta)=-\underline{d_{R}}(\theta)=$ $\left(|\cos \theta+\sin \theta \tan \theta| \dot{\omega}_{x}^{\max }, 0,|\tan \theta| \dot{\omega}_{x}^{\max }, 0\right)^{T}$, where $\dot{\omega}_{x}^{\max }$ is the maximum value for $\left|\dot{\omega}_{x}\right|$.

Remark 11: It is worth stating that the matrix $R(\vartheta)$ given by (69) depends on the state variables $u$ and $w$ through the angle-of-attack $\alpha$. Consequently, the matrix function $\dot{R}$ will depend on $\dot{u}$ and $\dot{w}$, which are not measured, in contrast with the assumption made in Section 4 that $\dot{\vartheta}$ is known. However, since it has been noticed that the elements depending on $\dot{u}$ and $\dot{w}$ are small in size, $\dot{R}$ can be approximated successfully by a matrix $\tilde{R}$, obtained from $R(\vartheta)$ assuming a constant $\alpha$, which depends only on measured variables, such that the proposed technique can still be applied despite not measuring $\dot{\vartheta}$.

\section{SIMULATION RESULTS}

The simulation results shown in this section have been obtained assuming that each parameter $C_{i}$ is affected by a symmetric uncertainty $\Delta C_{i}$ with bounds corresponding to $0.4 \%$ of the nominal value $\bar{C}_{i}$. Dryden-like wind disturbances [58], with $\dot{\omega}_{x}, \dot{\omega}_{z} \in\left[-0.1 \mathrm{~m} / \mathrm{s}^{2}, 0.1 \mathrm{~m} / \mathrm{s}^{2}\right]$ have been used to simulate the components of the wind gusts. It is assumed that the noise affecting the sensor measurements is uniformly distributed within the intervals $v_{u} \in[-1 \mathrm{~m} / \mathrm{s}, 1 \mathrm{~m} / \mathrm{s}], v_{w} \in[-1 \mathrm{~m} / \mathrm{s}, 1 \mathrm{~m} / \mathrm{s}], v_{q} \in$ $[-0.03 \mathrm{rad} / \mathrm{s}, 0.03 \mathrm{rad} / \mathrm{s}]$ and $v_{\theta} \in[-0.3 \mathrm{rad}, 0.3 \mathrm{rad}]$, where $v_{x}$ denotes the measurement noise for the state variable $x$. However, the raw measurements coming from the sensors have been filtered using low-pass filters with time constant $\tau=0.23 \mathrm{~s}$, and the filtered signals have been used as $y$ in (2), such that $V=[0.25,0.25,0.01,0.01]^{T}$.

In order to calculate the matrices appearing in (13)-(14), (16)-(17) and (41)-(44), let us notice that an optimal choice of the diagonal matrix functions $\underline{\Gamma}(\vartheta), \bar{\Gamma}(\vartheta)$ can be performed by maximizing the icing to wind/noise ratios (IWNRs) [24] for each residual. This choice enhances the residuals' ability to reject the wind acceleration disturbance and the noise, and increases their sensitivity to the icing. Also, due to the structure of the matrix $S$ in (68), the matrix functions $\underline{S}^{*}(\vartheta), \bar{S}^{*}(\vartheta)$ should be such that $\underline{F}(\vartheta), \bar{F}(\vartheta)$ are upper triangular, with the upper left diagonal block corresponding to $\underline{\Gamma}(\vartheta)$ and $\bar{\Gamma}(\vartheta)$, respectively. For the sake of simplicity, $\underline{S}^{*}(\vartheta)$ and $\bar{S}^{*}(\vartheta)$ can be chosen in such a way that diagonal matrix functions $\underline{F}(\vartheta)$ and $\bar{F}(\vartheta)$ are obtained. In this case, it is easy to ensure that the matrix functions $\underline{F}(\vartheta)$ and $\bar{F}(\vartheta)$ are Metzler.

The set of conditions given by (45) has been verified using 256 gridding points, which correspond to the partition of each interval of variation of the state variables $(u \in[16,21], w \in[0.5,2.5]$, $q \in[-0.003,0.003], \theta \in[-0.1,0.3])$ in 4 sub-intervals. 
For simulation purposes, the UAV is controlled by an autopilot [59], responsible of tracking some desired time-varying reference profiles for the horizontal velocity $u$ and the pitch angle $\theta$ (the calculated control inputs are illustrated in Fig. 2). Four different scenarios have been considered, as follows:

\section{Scenario 1}

In this scenario, no faults/icing occur. Throughout the simulation, the filtered measurements are always inside the estimated bounds, as shown in Fig. 3. As a consequence, the residuals obtained in this scenario are all positive (see Fig. 4), which means that the diagnosis algorithm given in Section 5 provides a no fault/no icing indication.

\section{Scenario 2}

The propulsion is subject to a linearly incipient loss of efficiency which starts at time $t=200 \mathrm{~s}$ and equals $\varphi_{t}(t)=0.5$ starting from time $t=210 \mathrm{~s}$. Fig. 5 shows that due to the fault occurrence, the filtered measurements exits from the estimated bounds, such that at time $t=202.7 \mathrm{~s}, \underline{\varepsilon}^{(1)}$ becomes negative (see Fig. 6), and since all the other residuals remain positive, a correct indication of fault in thrust is provided by the diagnosis algorithm.

\section{Scenario 3}

The elevator is subject to a linearly incipient loss of efficiency which starts at time $t=200 \mathrm{~s}$ and equals $\varphi_{e}(t)=0.9$ starting from time $t=210 \mathrm{~s}$. Fig. 7 shows that after the fault occurrence, the filtered measurements of both $u(t)$ and $q(t)$ exit the estimated bounds. However, due to the decoupling property of the residuals, only $\bar{\varepsilon}^{(2)}$ becomes negative (see Fig. 8), which allows the diagnosis algorithm to provide a correct indication of fault in elevator at time $t=204.62 \mathrm{~s}$.

\section{Scenario 4}

The UAV is subject to icing, i.e. the stability and control derivatives are modified according to (67), taking into account the coefficients $K_{i}$ listed in Table $\mathrm{II}^{\S}$. The icing starts at time $t=200 \mathrm{~s}$ and slowly increases $\eta$ from 0 to 0.2 , such that $\eta=0.2$ starting from time $t=400 \mathrm{~s}$. Fig. 9 shows that this scenario affects all the interval estimations of the states, except the ones corresponding to the pitch angle $\theta(t)$. On the other hand, the residuals plotted in Fig. 10 show that an abnormal situation is detected at time $t=275.06 s\left(\bar{\varepsilon}^{(3)}\right.$ becomes negative) and the icing occurrence is correctly isolated at time $t=285.04 s\left(\bar{\varepsilon}^{(2)}\right.$ becomes negative). Further confirmation about the icing occurrence is provided by the residual $\underline{\varepsilon}^{(1)}$, which becomes negative at time $t=322.36 \mathrm{~s}$.

\footnotetext{
$\S$ The coefficients $K_{i}$ used in this work have been computed mimicking the proportional variation of the stability and control derivatives for a Twin Otter aircraft subject to all iced condition [57], and they could differ in the case of a real Zagi Flying Wing UAV. However, since the proposed LPV interval unknown input observer does not depend on the values of these coefficients, it can be expected that similar results would be obtained with different values of the coefficients $K_{i}$. 

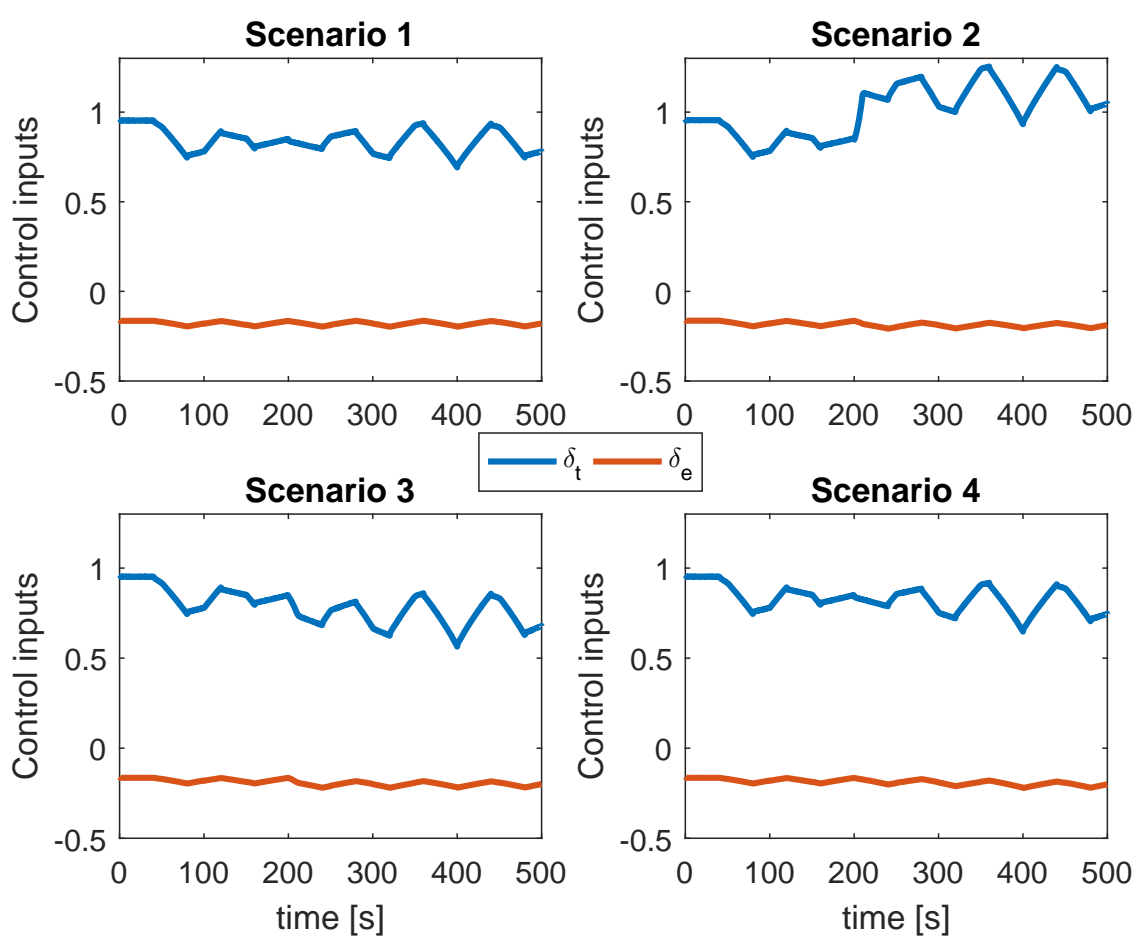

Figure 2. Control inputs $\delta_{t}(t)$ and $\delta_{e}(t)$ in scenarios 1-4.
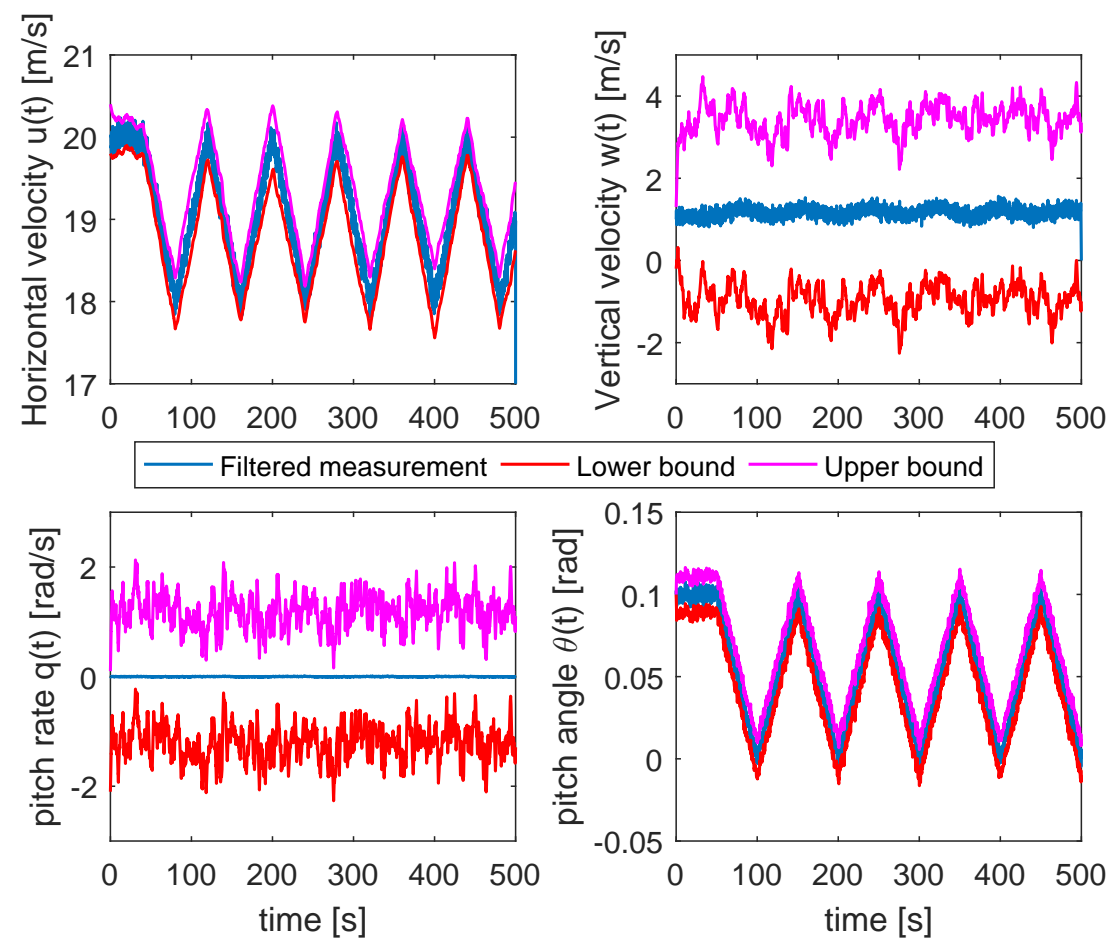

Figure 3. Filtered measurements and estimated bounds in scenario 1 (no fault/icing). 

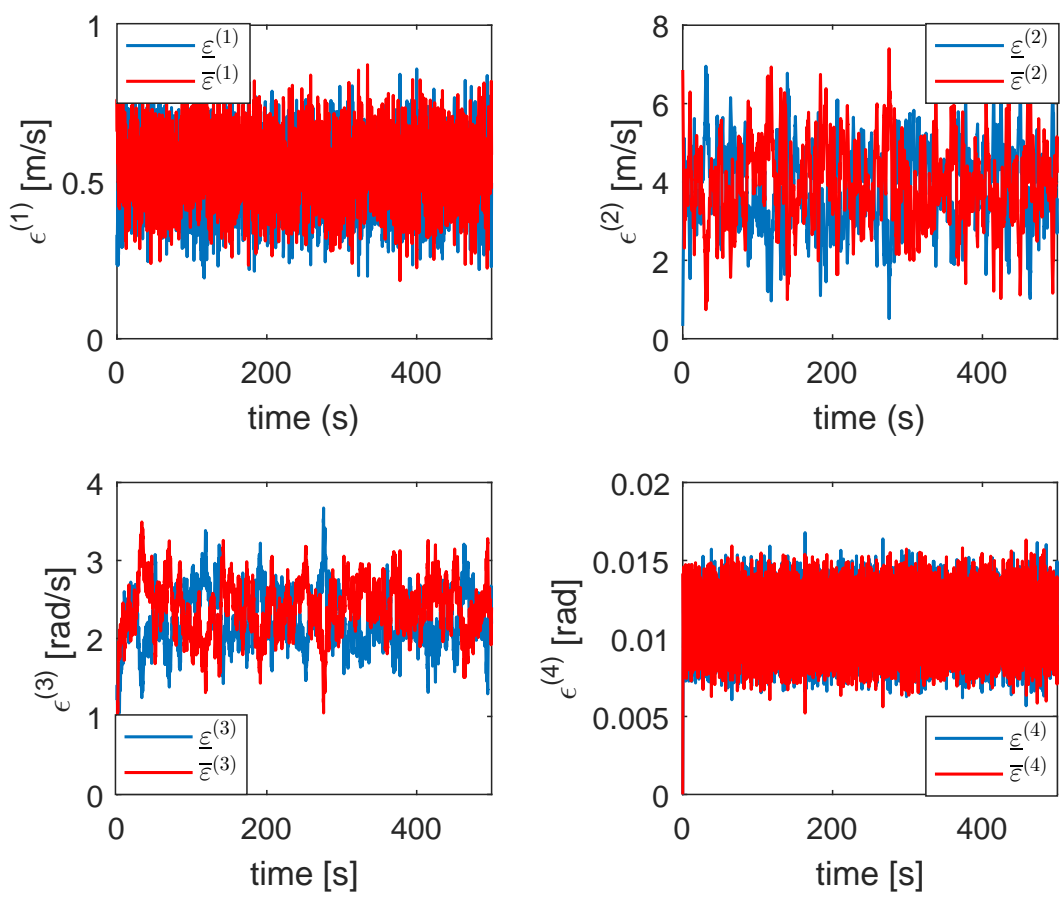

Figure 4. Residuals in scenario 1 (no fault/icing).
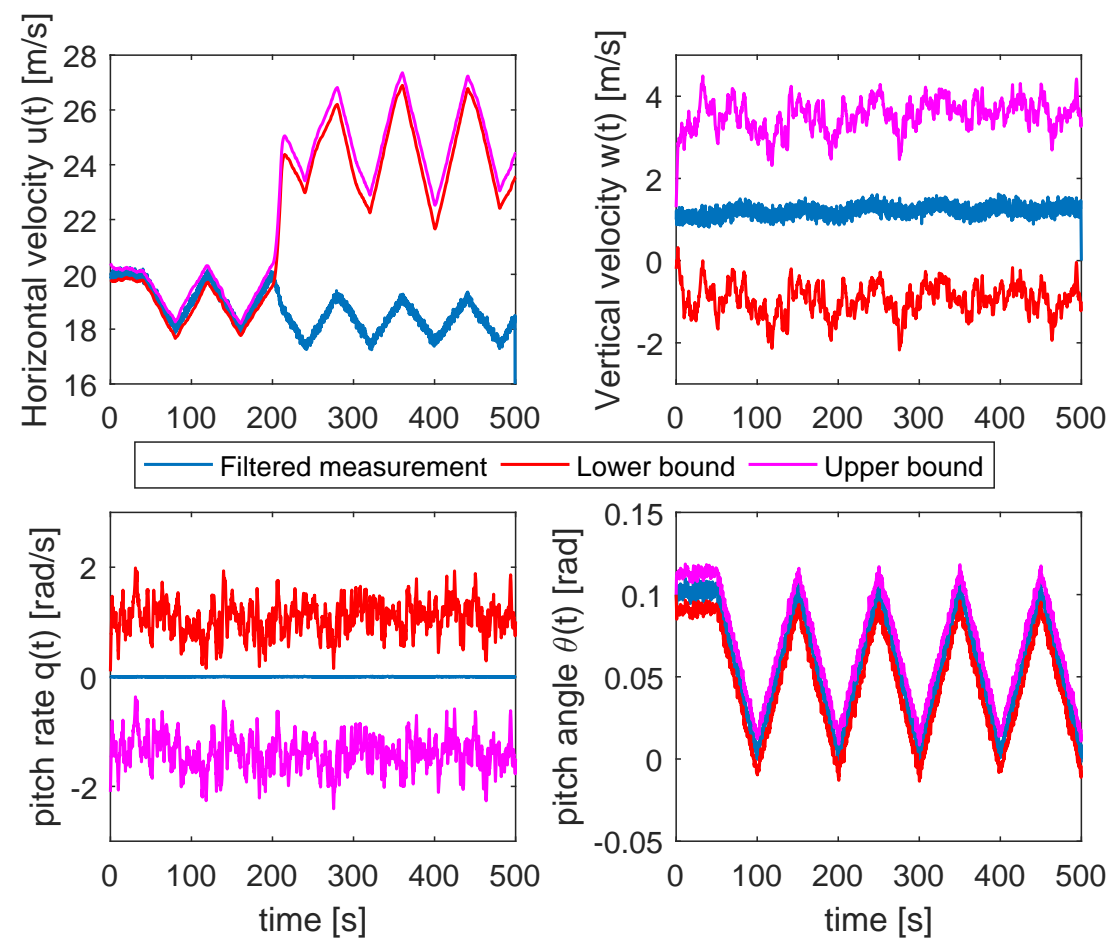

Figure 5. Filtered measurements and estimated bounds in scenario 2 (fault in thrust). 

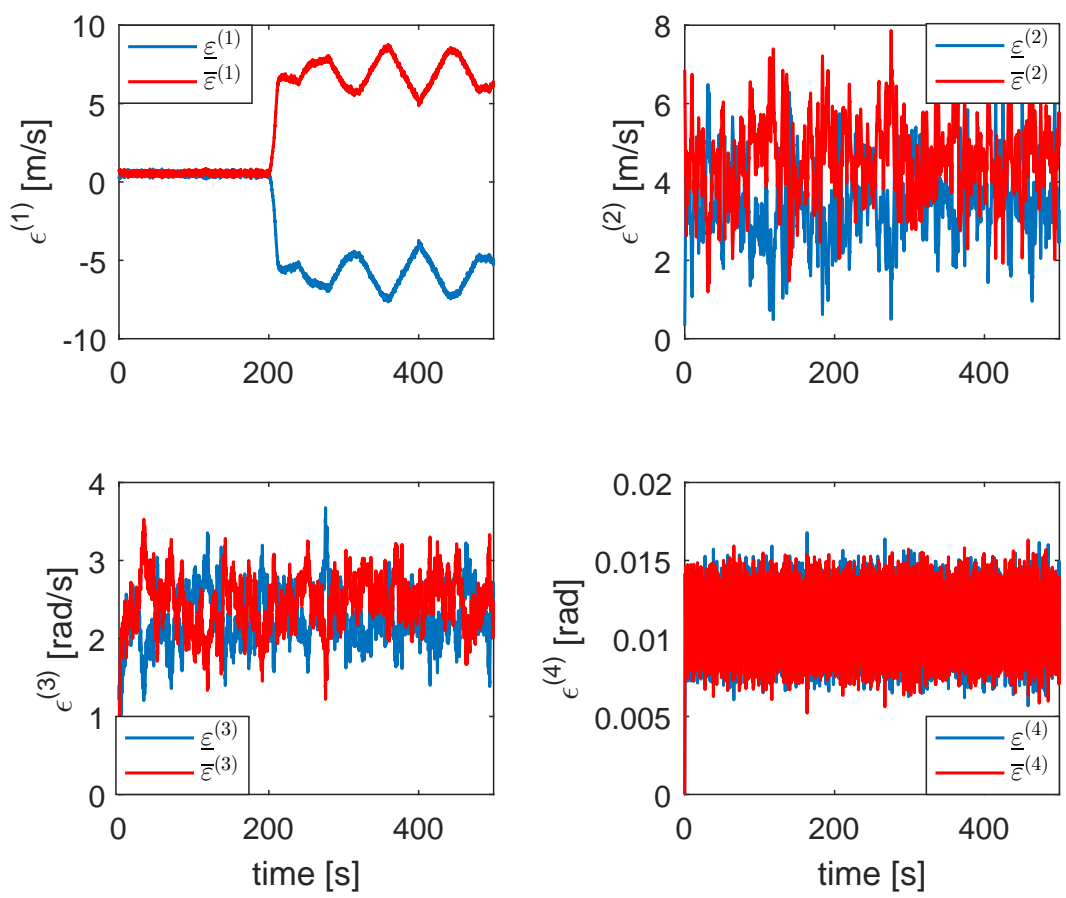

Figure 6. Residuals in scenario 2 (fault in thrust).
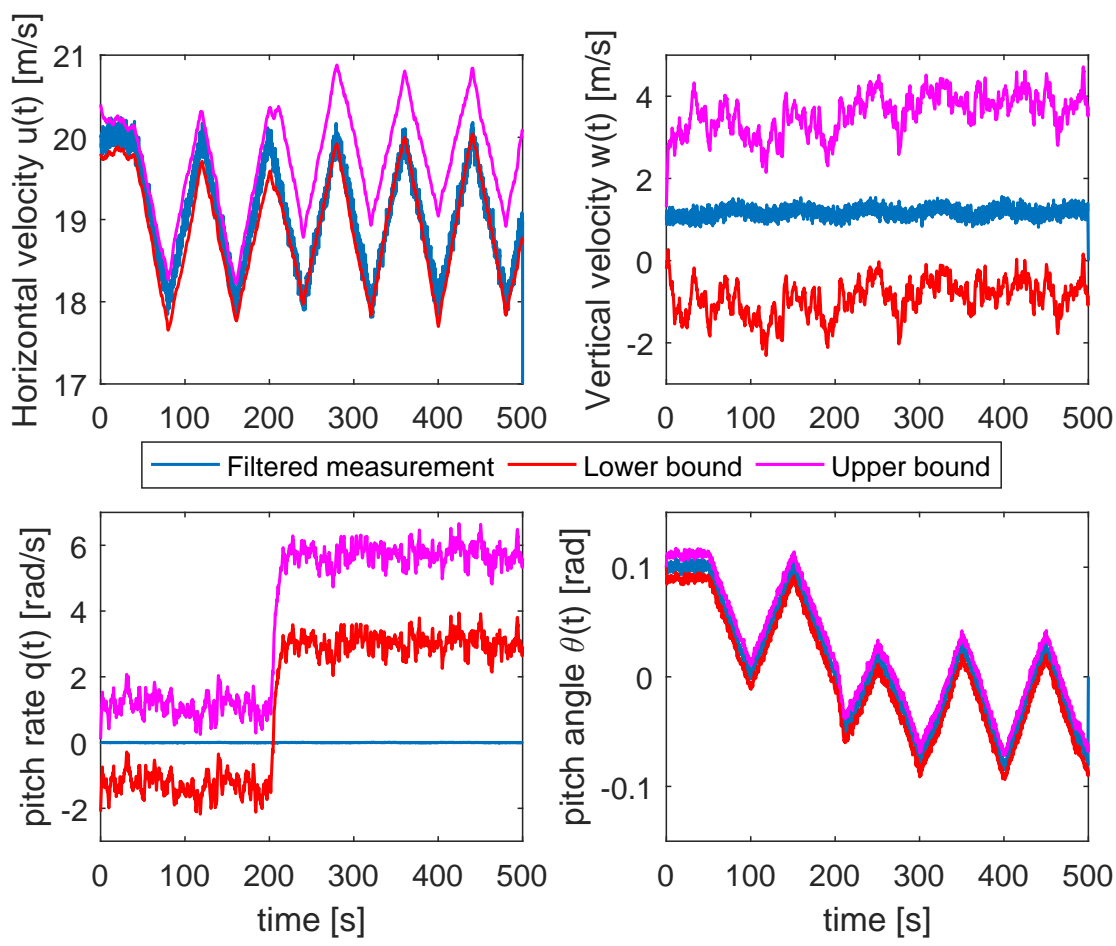

Figure 7. Filtered measurements and estimated bounds in scenario 3 (fault in elevator). 

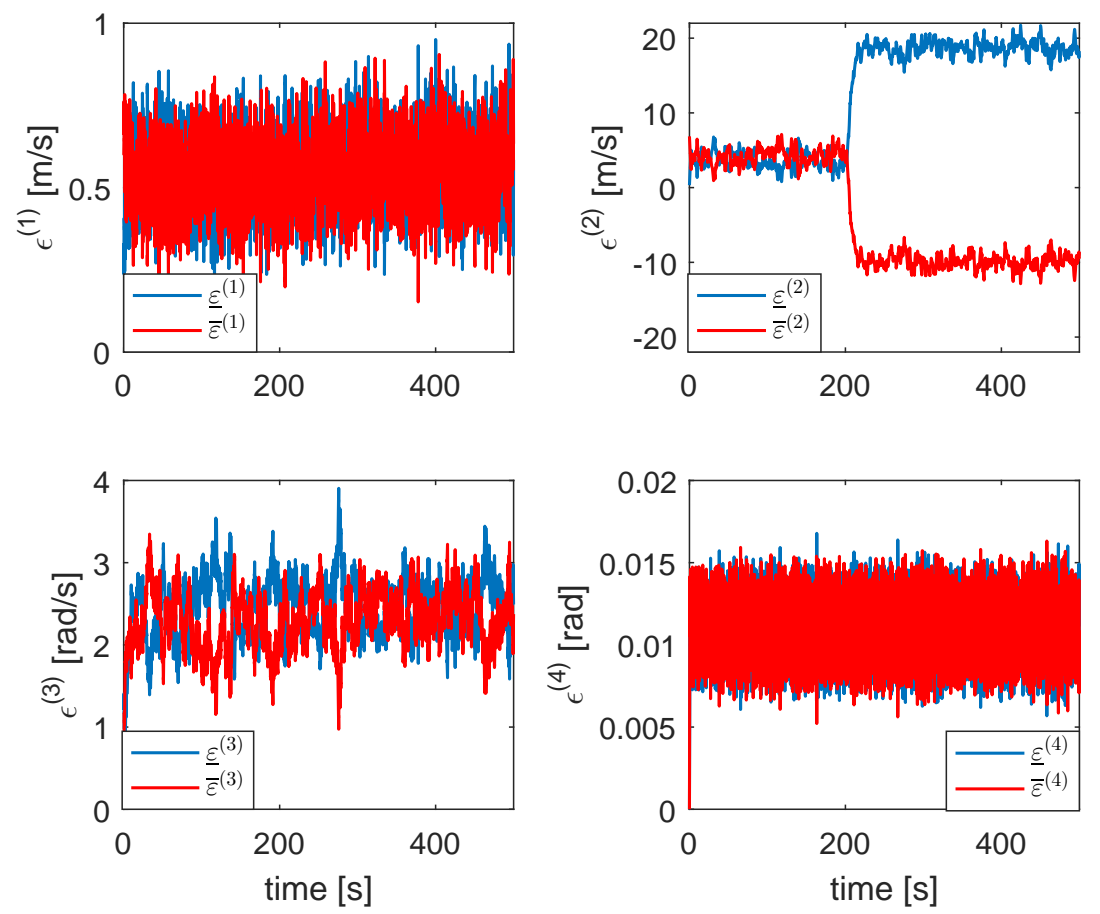

Figure 8. Residuals in scenario 3 (fault in elevator).
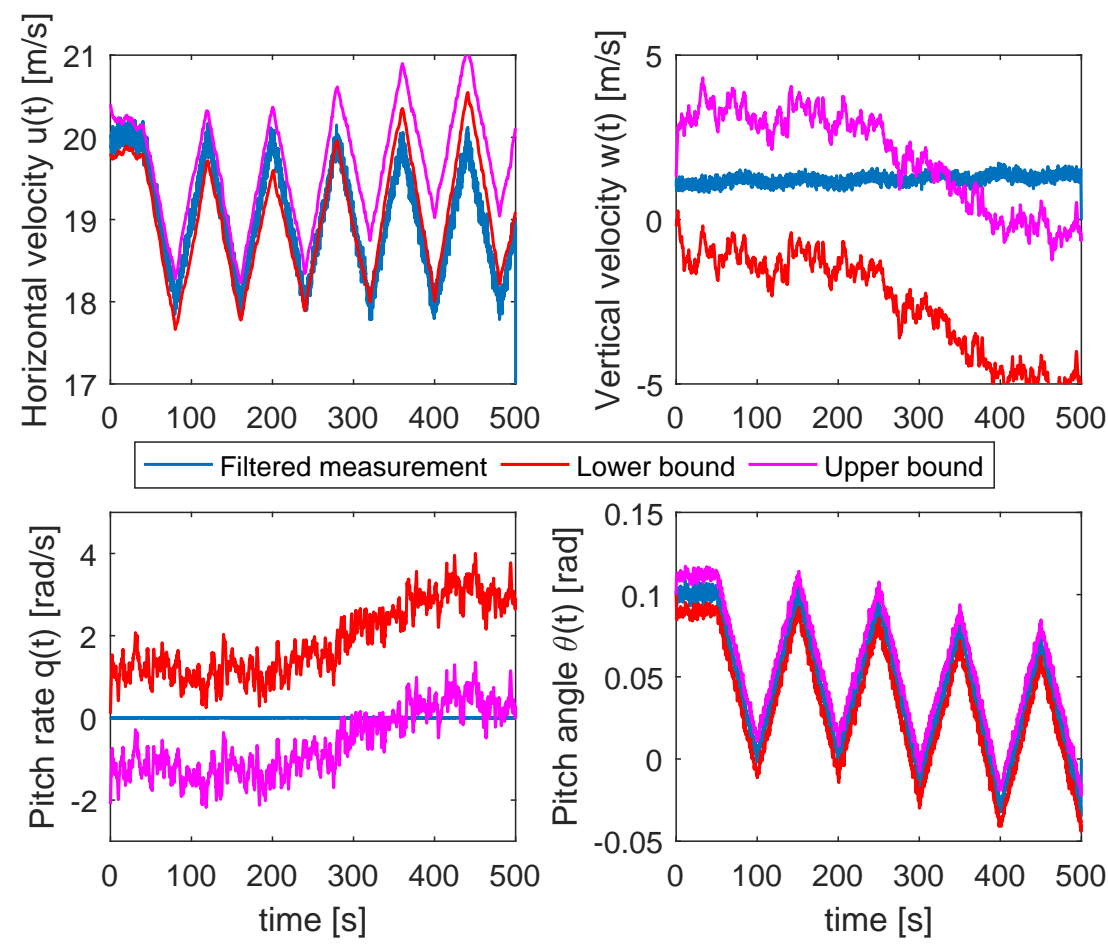

Figure 9. Filtered measurements and estimated bounds in scenario 4 (icing). 

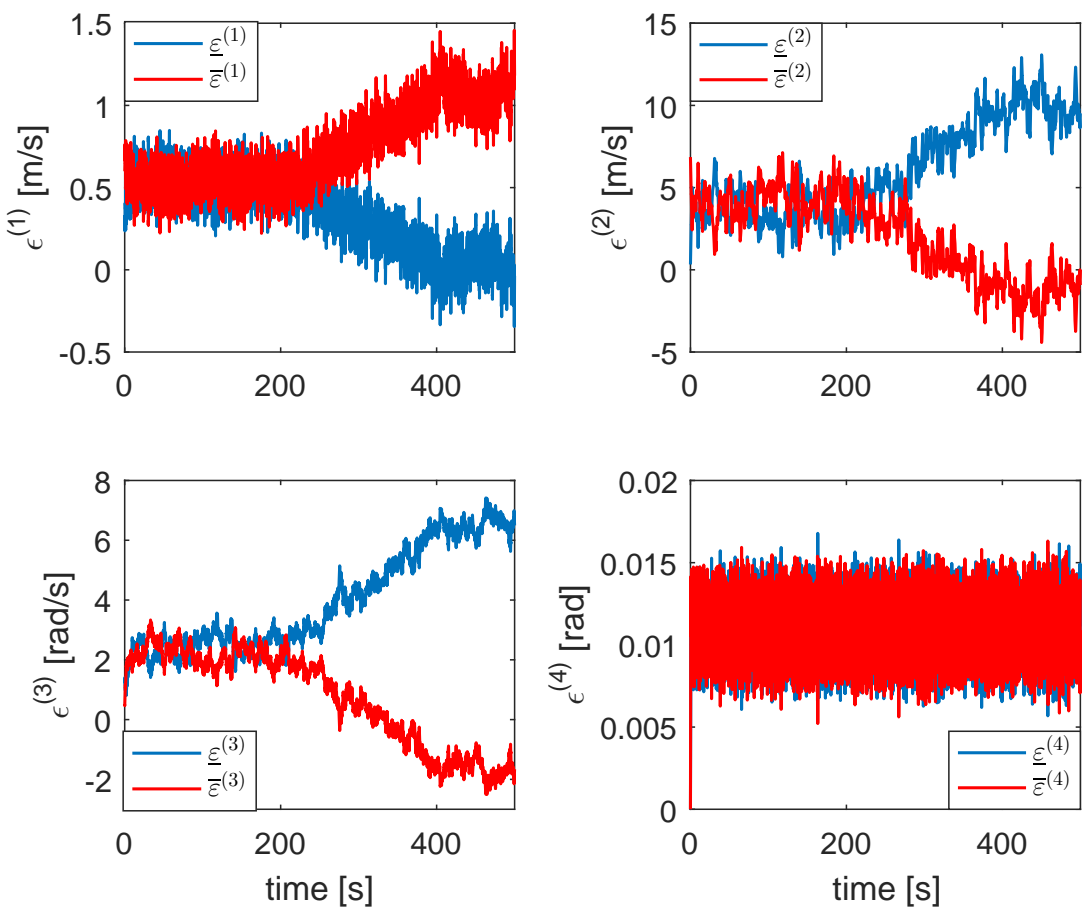

Figure 10. Residuals in scenario 4 (icing).

Table II. Coefficients $K_{i}$ for an all iced configuration

\begin{tabular}{cccccc}
\hline Coeff. & Value & Coeff. & Value & Coeff. & Value \\
\hline$K_{L_{0}}$ & 0 & $K_{L_{\alpha}}$ & -0.5000 & $K_{L_{q}}$ & -0.0675 \\
$K_{L_{\delta_{e}}}$ & -0.4770 & $K_{D_{0}}$ & 2.5610 & $K_{D_{\alpha}}$ & 0 \\
$K_{D_{q}}$ & 0 & $K_{D_{\delta_{e}}}$ & 0 & $K_{m_{0}}$ & 0 \\
$K_{m_{\alpha}}$ & -0.4960 & $K_{m_{q}}$ & -0.1755 & $K_{m_{\delta_{e}}}$ & -0.5000 \\
\hline
\end{tabular}

Remark 12: The main drawback of applying an interval strategy for dealing with uncertainties, noise and disturbances, is the inherent conservativeness due to the fact that the estimated bounds for the state will take into account the worst-case scenario. It has been observed through simulations that the theoretical assumptions under which the method has been developed can be relaxed in order to deal with larger values of uncertainties, noise and disturbances, although in this case no theoretical guarantees of false alarms avoidance can be provided.

\section{CONCLUSIONS}

This paper has proposed an LPV proportional integral interval UIO for the robust fault/icing detection in UAVs described by an uncertain model. The proposed technique has several advantages. 
First, it can take into account operating point variations in an elegant way using the LPV paradigm. Second, the presence of the integral term avoids the appearance of the noise derivative term in the estimation error equation, thus increasing the noise rejection properties. Third, due to the property of interval estimation guaranteed by the observer, as long as the assumptions about disturbances, noise and uncertainties hold, the absence of false alarms will be assured.

The conditions for the analysis and design of these observers are based on LMIs, which can be solved efficiently using available solvers. In particular, two properties are required by the analysis/design: i) interval estimation of the state, i.e., as long as some assumptions about bounds on uncertainties, disturbances and noise are verified, the state will always be contained within the bounds calculated by the interval observer; and ii) boundedness of the estimation, which is akin to the asymptotic stability of classical state observers, and is verified by finding an appropriate Lyapunov function.

Simulation results, obtained with the uncertain model of a Zagi Flying Wing UAV, have shown the effectiveness of the decision algorithm, which identifies correctly unexpected changes in the system dynamics due to actuator faults or icing. Four scenarios have given more insight into the proposed method and have confirmed the results provided by the theory.

Future research will be aimed at decreasing the conservativeness of the proposed method, both by using alternative analysis/design approaches based on less conservative Lyapunov functions (e.g. parameter-dependent ones) and by relaxing the assumptions on disturbances and noise. Another line of research will investigate the active choice of the reference trajectory in order to enhance the diagnosis performance of the proposed method. It is worth recalling that a theoretical challenge to be considered in the future lies in extending the proposed method to the more general case in which the output matrix depends on the varying parameters. Finally, the experimental validation of the proposed methodology will be pursued.

\section{ACKNOWLEDGMENTS}

This work has been supported by a grant from Iceland, Liechtenstein and Norway through the EEA Financial Mechanism. Operated by Universidad Complutense de Madrid (ref. 006-ABEL-IM2014B). The authors also acknowledge support by the Spanish Government (MINECO) and FEDER through the project CICYT HARCRICS (ref. DPI2014-58104-R), by the DGR of the Generalitat de Catalunya (ref. 2017/SGR/482), by the Spanish State Research Agency through the María de Maeztu Seal of Excellence to IRI (ref. MDM-2016-0656) and the grant Juan de la Cierva Formación (ref. FJCI-2016-29019), and by the Research Council of Norway through the Centres of Excellence funding scheme (ref. 223254 - AMOS). D. Rotondo has been also supported by the ERCIM Alain 
Bensoussan Fellowship programme. The authors would like to thank Dr. Helem Sabina Sánchez for improving the rendering of Fig. 1.

\section{APPENDIX}

$$
\begin{aligned}
& \bar{a}_{11}(\cdot)=\frac{\rho u S}{2 m}\left[\left(\bar{C}_{L_{0}}+\bar{C}_{L_{\alpha}} \alpha\right) \sin \alpha-\left(\bar{C}_{D_{0}}+\bar{C}_{D_{\alpha}} \alpha\right) \cos \alpha-\frac{S_{\text {prop }} C_{\text {prop }}}{S}\right] \\
& \bar{a}_{12}(\cdot)=\frac{\rho w S}{2 m}\left[\left(\bar{C}_{L_{0}}+\bar{C}_{L_{\alpha}} \alpha\right) \sin \alpha-\left(\bar{C}_{D_{0}}+\bar{C}_{D_{\alpha}} \alpha\right) \cos \alpha-\frac{S_{\text {prop }} C_{\text {prop }}}{S}\right] \\
& \bar{a}_{13}(\cdot)=-w+\frac{\rho S c V_{a}}{4 m}\left(\bar{C}_{L_{q}} \sin \alpha-\bar{C}_{D_{q}} \cos \alpha\right) \\
& \bar{a}_{21}(\cdot)=-\frac{\rho u S}{2 m}\left[\left(\bar{C}_{D_{0}}+\bar{C}_{D_{\alpha}} \alpha\right) \sin \alpha+\left(\bar{C}_{L_{0}}+\bar{C}_{L_{\alpha}} \alpha\right) \cos \alpha\right] \\
& \bar{a}_{22}(\cdot)=-\frac{\rho w S}{2 m}\left[\left(\bar{C}_{D_{0}}+\bar{C}_{D_{\alpha}} \alpha\right) \sin \alpha+\left(\bar{C}_{L_{0}}+\bar{C}_{L_{\alpha}} \alpha\right) \cos \alpha\right] \\
& \bar{a}_{23}(\cdot)=u-\frac{\rho S c V_{a}}{4 m}\left(\bar{C}_{D_{q}} \sin \alpha+\bar{C}_{L_{q}} \cos \alpha\right) \\
& \bar{a}_{31}(\cdot)=\frac{\rho S c u}{2 J_{y}}\left(\bar{C}_{m_{0}}+\bar{C}_{m_{\alpha}} \alpha\right) \\
& \bar{a}_{32}(\cdot)=\frac{\rho S c w}{2 J_{y}}\left(\bar{C}_{m_{0}}+\bar{C}_{m_{\alpha}} \alpha\right) \\
& \bar{a}_{33}(\cdot)=\frac{\rho V_{a} S c^{2}}{4 J_{y}} \bar{C}_{m_{q}} \\
& b_{11}=\frac{\rho S_{\text {prop }} C_{\text {prop }}}{2 m} k_{m}^{2} \\
& \bar{b}_{12}(\cdot)=\frac{\rho S V_{a}^{2}}{2 m}\left(\bar{C}_{L_{\delta_{e}}} \sin \alpha-\bar{C}_{D_{\delta_{e}}} \cos \alpha\right) \\
& \bar{b}_{22}(\cdot)=-\frac{\rho V_{a}^{2} S}{2 m}\left(\bar{C}_{D_{\delta_{e}}} \sin \alpha+\bar{C}_{L_{\delta_{e}}} \cos \alpha\right) \\
& \bar{b}_{32}(\cdot)=\frac{\rho V_{a}^{2} S c}{2 J_{y}} \bar{C}_{m_{\delta_{e}}} \\
& \Delta a_{11}(\cdot)=\frac{\rho u S}{2 m}\left[\left(\Delta C_{L_{0}}+\Delta C_{L_{\alpha}} \alpha\right) \sin \alpha-\left(\Delta C_{D_{0}}+\Delta C_{D_{\alpha}} \alpha\right) \cos \alpha\right] \\
& \Delta a_{12}(\cdot)=\frac{\rho w S}{2 m}\left[\left(\Delta C_{L_{0}}+\Delta C_{L_{\alpha}} \alpha\right) \sin \alpha-\left(\Delta C_{D_{0}}+\Delta C_{D_{\alpha}} \alpha\right) \cos \alpha\right] \\
& \Delta a_{13}(\cdot)=\frac{\rho S c V_{a}}{4 m}\left(\Delta C_{L_{q}} \sin \alpha-\Delta C_{D_{q}} \cos \alpha\right) \\
& \Delta a_{21}(\cdot)=-\frac{\rho u S}{2 m}\left[\left(\Delta C_{D_{0}}+\Delta C_{D_{\alpha}} \alpha\right) \sin \alpha+\left(\Delta C_{L_{0}}+\Delta C_{L_{\alpha}} \alpha\right) \cos \alpha\right] \\
& \Delta a_{22}(\cdot)=-\frac{\rho w S}{2 m}\left[\left(\Delta C_{D_{0}}+\Delta C_{D_{\alpha}} \alpha\right) \sin \alpha+\left(\Delta C_{L_{0}}+\Delta C_{L_{\alpha}} \alpha\right) \cos \alpha\right] \\
& \Delta a_{23}(\cdot)=\frac{\rho S c V_{a}}{4 m}\left(\Delta C_{D_{q}} \sin \alpha+\Delta C_{L_{q}} \cos \alpha\right)
\end{aligned}
$$




$$
\begin{gathered}
\Delta a_{31}(\cdot)=\frac{\rho S c u}{2 J_{y}}\left(\Delta C_{m_{0}}+\Delta C_{m_{\alpha}} \alpha\right) \\
\Delta a_{32}(\cdot)=\frac{\rho S c w}{2 J_{y}}\left(\Delta C_{m_{0}}+\Delta C_{m_{\alpha}} \alpha\right) \\
\Delta a_{33}(\cdot)=\frac{\rho V_{a} S c^{2}}{4 J_{y}} \Delta C_{m_{q}} \\
\Delta b_{12}(\cdot)=\frac{\rho S V_{a}^{2}}{2 m}\left(\Delta C_{L_{\delta_{e}}} \sin \alpha-\Delta C_{D_{\delta_{e}}} \cos \alpha\right) \\
\Delta b_{22}(\cdot)=-\frac{\rho V_{a}^{2} S}{2 m}\left(\Delta C_{D_{\delta_{e}}} \sin \alpha+\Delta C_{L_{\delta_{e}}} \cos \alpha\right) \\
\Delta b_{32}(\cdot)=\frac{\rho V_{a}^{2} S c}{2 J_{y}} \Delta C_{m_{\delta_{e}}}
\end{gathered}
$$

\section{REFERENCES}

[1] Zhang Y, Jiang J. Bibliographical review on reconfigurable fault-tolerant control systems. Annual Reviews in Control 2008; 32:229-252.

[2] Gao Z, Cecati C, Ding SX. A survey of fault diagnosis and fault-tolerant techniques - Part I: fault diagnosis with model-based and signal-based approaches. IEEE Transactions on Industrial Electronics 2015; 62(6):3757-3767.

[3] De Persis C, Isidori A. A geometric approach to nonlinear fault detection and isolation. IEEE Transactions on Automatic Control 2001; 46:853-865.

[4] Kaboré P, Wang H. Design of fault diagnosis filters and fault-tolerant control for a class of nonlinear systems. IEEE Transactions on Automatic Control 2001; 46:1805-1810.

[5] Lu P, Van Eykeren L, Van Kampen E, Chu QP. Selective-reinitialization multiple-model adaptive estimation for fault detection and diagnosis. Journal of Guidance, Control, and Dynamics 2015; 38:1409-1424.

[6] Caliskan F, Hajijev C. A review of in-flight detection and identification of aircraft icing and reconfigurable control. Progress in Aerospace Sciences 2013; 60:12-34.

[7] Gent R, Dart N, Cansdale J. Aircraft icing. Phil. Trans. of the Royal Soc. of London, Series A: Mathematical, Physical and Engineering Sciences 2000; 358:2873-2911.

[8] Rutherford R, Dudman R. Zoned aircraft de-icing system and method Oct 1999. URL http: //www.google.com/patents/US6237874. 
[9] Sørensen KL, Helland AS, Johansen TA. Carbon nanomaterial-based wing temperature control system for in-flight anti-icing and de-icing of unmanned aerial vehicles. IEEE Aerospace Conference, 2015.

[10] Sørensen KL, Johansen TA. Thermodynamics of a carbon nano-materials based icing protection system for unmanned aerial vehicle. IEEE Aerospace Conference, 2016.

[11] Hajijev C, Caliskan F. Fault diagnosis and reconfiguration in flight control systems. Springer Science and Business Media, 2003.

[12] Cristofaro A, Johansen TA, Aguiar AP. Icing detection and identification for unmanned aerial vehicles: multiple model adaptive estimation. Proc. of the European Control Conference (ECC), 2015; 1651-1656.

[13] Seron MM, Johansen TA, Doná JAD, Cristofaro A. Detection and estimation of icing in unmanned aerial vehicles using a bank of unknown input observers. Proc. of the 5th Australian Control Conference (UACC), 2015; 87-92.

[14] Sørensen KL, Blanke M, Johansen TA. Diagnosis of wing icing through lift and drag coefficient change detection for small unmanned aircraft. Proc. of the 9th IFAC Symposium on Fault Detection, Supervision and Safety of Technical Processes, 2015; 541-546.

[15] Wenz A, Johansen TA. Icing detection for small fixed wing UAVs using inflight aerodynamic coefficient estimation. Proc. of the IEEE Conference on Control and Applications (CCA), $2016 ; 230-236$.

[16] Armanini SF, Polak M, Gantrey IE, Lucas A, Whidborne JF. Decision-making for unmanned aerial vehicle operation in icing conditions. CEAS Aeronautical Journal 2016; 7(4):663-675.

[17] Hammouri H, Tmar Z. Unknown input observer for state affine systems: a necessary and sufficient condition. Automatica 2010; 46:271-278.

[18] Chen J, Patton RJ, Zhang HY. Design of unknown input observers and robust fault-detection filters. Internacional Journal of Control 1996; 63(1):85-105.

[19] Cristofaro A, Johansen TA. Fault tolerant control allocation using unknown input observers. Automatica 2014; 50:1891-1897.

[20] Tousi MM, Khorasani K. Robust observer-based fault diagnosis for an unmanned aerial vehicle. Proc. of the IEEE International Systems Conference (SysCon), 2011; 428-434.

[21] Cristofaro A, Johansen TA. An unknown input observer approach to icing detection for unmanned aerial vehicles with linearized longitudinal motion. Proc. of the American Control Conference (ACC), 2015; 207-213. 
[22] Cristofaro A, Johansen TA. An unknown input observer based control allocation scheme for icing diagnosis and accommodation in overactuated UAVs. Proc. of the European Control Conference (ECC), 2016.

[23] Rotondo D, Cristofaro A, Johansen TA, Nejjari F, Puig V. Icing detection in unmanned aerial vehicles with longitudinal motion using an LPV unknown input observer. Proc. of the 9th IEEE Multiconference on Systems and Control, 2015; 984-989.

[24] Rotondo D, Cristofaro A, Johansen TA, Nejjari F, Puig V. Detection of icing and actuator faults in the longitudinal dynamics of small UAVs using an LPV proportional integral unknown input observer. Proc. of the 3rd Conference on Control and Fault-Tolerant Systems (SysTol), 2016.

[25] Chebotarev S, Efimov D, Raïssi T, Zolghadri A. On interval observer design for a class of continuous-time LPV systems. Proceedings of the 9th IFAC Symposium on Nonlinear Control Systems, 2013; 68-73.

[26] Efimov D, Raïssi T, Perruquetti W, Zolghadri A. Estimation and control of discrete-time LPV systems using interval observers. Proceedings of the IEEE 52nd Annual Conference on Decision and Control (CDC), 2013; 5036-5041.

[27] Wang Y, Bevly DM, Rajamani R. Interval observer design for LPV systems with parametric uncertainty. Automatica 2015; 60:79-85.

[28] Gouzé JL, Rapaport A, Hadj-Sadok MZ. Interval observers for uncertain biological systems. Ecological Modeling 2000; 133:46-56.

[29] Simon D. Optimal state estimation. John Wiley and Sons, 2006.

[30] Raka S, Combastel C. Fault detection based on robust adaptive thresholds: a dynamic interval approach. Annual Reviews in Control 2013; 37(1):119-128.

[31] Olivier B, Gouzè J. Closed loop observers bundle for uncertain biotechnological models. Journal of Process Control 2004; 14(7):765-774.

[32] Moisan M, Bernard O, Gouzè J. Near optimal interval observers bundle for uncertain bioreactors. Automatica 2009; 45(1):291-295.

[33] Raïssi T, Videan G, Zolghadri A. Interval observers design for consistency checks of nonlinear continuous-time systems. Automatica 2010; 46(3):518-527.

[34] Raïssi T, Efimov D, Zolghadri A. Interval state estimation for a class of nonlinear systems. IEEE Transactions on Automatic Control 2012; 57(1):260-265. 
[35] Rotondo D, Cristofaro A, Johansen TA, Nejjari F, Puig V. State estimation and decoupling of unknown inputs in uncertain LPV systems using interval observers. International Journal of Control 2017; .

[36] Penrose R. A generalized inverse for matrices. Mathematical Proceedings of the Cambridge Philosophical Society 1955; 51(3):406-413.

[37] Apkarian P, Gahinet P, Becker G. Self-scheduled $H_{\infty}$ Control of Linear Parameter-Varying Systems: A Design Example. Automatica 1995; 31(9):1251 - 1261.

[38] Koenig D, Mammar S. Design of proportional-integral observers for unknown input descriptor systems. IEEE Transactions on Automatic Control 2002; 47(12):2057-2062.

[39] Koenig D. Unknown input proportional multiple-integral observer design for linear descriptor systems: application to state and fault estimation. IEEE Transactions on Automatic Control 2005; 50(2):212-217.

[40] Youssef T, Chadli M, Karimi HR, Zelmat M. Design of unknown inputs proportional integral observers for TS fuzzy models. Neurocomputing 2014; 123:156-165.

[41] Farina L, Rinaldi S. Positive linear systems: theory and applications. Wiley, New York, 2000.

[42] Sontag ED. Input to state stability: Basic concepts and results. Nonlinear and optimal control theory. Springer, 2008; 163-220.

[43] Löfberg J. YALMIP : a toolbox for modeling and optimization in MATLAB. Proceedings of the CACSD Conference, Taipei, Taiwan, 2004.

[44] Sturm JF. Using SeDuMi 1.02, a MATLAB toolbox for optimization over symmetric cones. Optimization Methods and Software 1999; 11-12:625-653.

[45] Rosa P, Balas GJ, Silvestre C, Athans M. A synthesis method of LTI MIMO robust controllers for uncertain LPV plants. IEEE Transactions on Automatic Control 2014; 59(8):2234-2240.

[46] Montes de Oca S, Puig V, Blesa J. Robust fault detection based on adaptive threshold generation using interval lpv observers. International Journal of Adaptive Control and Signal Processing 2012; 26(3):258-283.

[47] Zhong M, Ding SX, Lam J, Wang H. An LMI approach to design robust fault detection filter for uncertain LTI systems. Automatica 2003; 39(3):543-550.

[48] Puig V, de Oca SM, Blesa J. Adaptive threshold generation in robust fault detection using interval models: time-domain and frequency-domain approaches. International Journal of Adaptive Control and Signal Processing 2013; 27(10):873-901. 
[49] Beard RW, McLain TW. Small Unmanned Aircraft: Theory and Practice. Princeton, NJ, USA: Princeton University Press, 2012.

[50] Johansen TA, Cristofaro A, Sørensen KL, Hansen JM, Fossen TI. On estimation of wind velocity, angle-of-attack and sideslip angle of small UAVs using standard sensors. Proceedings of the International Conference on Unmanned Aircraft Systems (ICUAS), 2015; 510-519.

[51] Kwiatkowski A, Boll MT, Werner H. Automated Generation and Assessment of Affine LPV Models. Proceedings of the 45th IEEE Conference on Decision and Control, 2006; 6690-6695.

[52] Rotondo D, Puig V, Nejjari F, Witczak M. Automated generation and comparison of TakagiSugeno and polytopic quasi-LPV models. Fuzzy Sets and Systems 2015; 277:44-64.

[53] Shamma J, Athans M. Gain Scheduling: Potential Hazards and Possible Remedies. IEEE Control Systems Magazine 1992; 12:101-107.

[54] Tan W, Packard AK, Balas GJ. Quasi-LPV modeling and LPV control of a generic missile. Proceedings of the American Control Conference, 2000; 3692-3696.

[55] Andrés M, Balas GJ. Development of Linear Parameter Varying Models for Aircraft. Journal of Guidance, Control and Dynamics 2004; 27(2):218-228.

[56] Shin JY, Balas GJ, Kaya MA. Blending methodology of linear parameter varying control synthesis of f-16 aircraft system. Journal of Guidance, Control, and Dynamics 2002; 25(6):1040-1048.

[57] Bragg MB, Hutchinson T, Merret J, Oltman R, Pokhariyal D. Effect of ice accretion on aircraft flight dynamics. Proceedings of the 38th AIAA Aerospace Science Meeting and Exhibit, 2000.

[58] Hoblit FM. Gust loads on aircraft: concept and applications. American Institute of Aeronautics and Astronautics: Washington, DC, USA, 1988.

[59] Rotondo D, Cristofaro A, Gryte K, Johansen TA. LPV model reference control for fixed-wing uavs. IFAC-PapersOnLine 2017; 50(1):11 559-11 564. 